\title{
Model-based broadband estimation of cutting forces and tool vibration in milling through in-process indirect multiple-sensors measurements
}

ALBERTELLI, PAOLO; GOLETTI, MASSIMO; TORTA, MATTIA; SALEHI, MEHDI; MONNO, MICHELE

This is a post-peer-review, pre-copyedit version of an article published in INTERNATIONAL JOURNAL, ADVANCED MANUFACTURING TECHNOLOGY. The final authenticated version is available online at: http://dx.doi.org/10.1007/s00170-015-7402-x

This content is provided under CC BY-NC-ND 4.0 license

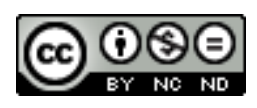




\title{
Model-based broadband estimation of cutting forces and tool vibration in milling through in-process indirect multiple-sensors measurements
}

\author{
P. Albertellia*, M. Goletti ${ }^{\mathrm{b}}$, M. Torta ${ }^{\mathrm{b}}$, M. Salehi ${ }^{\mathrm{b}}$, M. Monno ${ }^{\mathrm{a}}$ \\ ${ }^{a}$ Mechanical Engineering Department, Politecnico di Milano, Via La Masa 1, 20156 Milan (Italy) \\ ${ }^{b}$ Consorzio MUSP, Via Tirotti 9, 29122 Piacenza (Italy) \\ * Corresponding author. Tel.: +390223998557; fax: +390223998585. E-mail address: paolo.albertelli@polimi.it.
}

\begin{abstract}
In machining processes, cutting forces measurement is essential to allow cutting process and tool conditions monitoring. Moreover, in order to have information about the quality of the milled part, the amplitude of the tool tip vibration would be very useful. Since both the measurements are extremely complicated especially in an industrial scenario, in this study, an in-process model based estimator of cutting forces and tool tip vibration was designed and properly tested. The developed estimator relies on both a machine dynamic model and on indirect measurements coming from multiple sensors placed in the machine. The machine dynamic model was obtained through an experimental modal analysis session. The estimator was developed according to the Kalman Filter approach. The fusion of multiple sensors data allowed the compensation of machine tool dynamics over an extended frequency range. The accuracy of the observer estimations was checked performing two different experimental sessions in which both the force applied to tool and the tool tip vibration amplitude were measured. In the first session, the tool was excited with different sensorized hammers in order to appreciate the broad-bandwidth of the performed estimations. In the second one, real cutting tests (steel milling) were done and the cutting forces were measured through a dynamometer, tool tip vibrations were measured as well. The experimental results showed that the indirect estimation of cutting forces and tool tip vibrations exhibit a good agreement with respect to the corresponding measured quantities in low and high frequency ranges. The contribution of this research is twofold. Firstly, the conceived observer allows estimating the tool tip vibrations that is a useful information strictly connected to the surfaces quality of the processed workpiece. Secondly, thanks to a multi-sensors approach, the frequency bandwidth is extended especially in the low frequency range.
\end{abstract}

Keywords: Cutting force indirect estimation; Kalman Filter; Tool tip vibration estimation, Milling; Sensor Fusion

\section{Introduction}

Current industries demands attract manufacturer's attention on automation and high-quality machining. Condition monitoring of machine tools and processes allows improving the quality of products, the productivity and, in addition, it can insure an economic usage of machine tools [1]-[3].

Cutting forces, as some of the most important cutting process related indicators, can determine limits of cutting conditions, accuracy of the produced parts, machine tools and tools components failures, and other process information, which are useful for controlling and monitoring the machining processes [4]-[6].

In-process cutting forces measurement can be performed by dynamometers that are the most accurate transducers for the purpose. However, they are restricted to the laboratory use rather than to practical applications because of the limited size and mounting constraints, the high costs of the device, etc. In addition, the dynamic properties of dynamometric plates are strongly dependent on the workpieces inertia that depends on the specific processed part and can significantly change during the various machining phases. In order to overcome some of the mentioned problems, force sensors can be integrated into the spindle structure rather than installing them in the machine table. Smith et al. [7] and Aoyama et al. [8] reported research works in which rotating force and torque dynamometers were mounted, like adapters, between the spindle and the tool in order to locate the force sensors as close as possible to the cutting process. Nevertheless, the mentioned method reduces the dynamic properties of the spindle structure since the rotating force sensor has an excessive inertia. Alternative solutions that guaranteed a stiffer set-up used sensors mounted in the machine structure and a modelbased approach to improve cutting forces measurements. For instance, Altintas et al. [10] used piezo-electric load sensors mounted in the spindle housing, the measurements were processed by a Kalman filter in order to compensate the effects of structural flexibility on the force measurement. Albrecht et al. [9] developed an indirect method for measuring the cutting forces: it was based on the spindle shaft displacements measurements performed with capacitance sensors and on Kalman filtering processing. They also designed a setup based on three displacement sensors (placed at 120 degrees) suitable for two-dimensional forces (milling) indirect estimation and for the automatic temperature compensation. A similar work was also developed by Chang et al. [11]. The model used for the Kalman filtering was obtained analyzing the rotating spindle dynamic responses to excitations applied with a contactless magnetic device.

Matsubara et al. [12] studied cutting forces estimation by processing radial spindle shaft-housing relative displacements. For this purpose, four contamination-resistant eddy-current displacement sensors were used. In their 
research work, the calculation of the cutting forces from spindle displacement involved two major issues: thermal influence and spindle dynamic stiffness.

Over the years, several research works on cutting force estimation by indirect measurements technics have been carried out. Nonetheless, there are only few cases in which the estimation were investigated on the application of multiple sensors, Tonshoff and Inasaki [13].

\begin{tabular}{|c|c|c|c|}
\hline \multicolumn{4}{|c|}{ Nomenclature } \\
\hline$M$ & Mass matrix $[\mathrm{kg}]$ & $\alpha(s)_{j, k}$ & Dynamic compliance output $\mathrm{j}$, input $\mathrm{k}[\mathrm{m} / \mathrm{N}]$ \\
\hline $\boldsymbol{R}_{d}$ & Damping matrix $[\mathrm{Ns} / \mathrm{m}]$ & $X, Y, Z$ & Machine tool axes \\
\hline $\boldsymbol{K}_{s}$ & Stiffness matrix $[\mathrm{N} / \mathrm{m}]$ & $n_{x}, n_{y}$ & $\begin{array}{l}\text { Numbers of states of the model (X and } \mathrm{Y} \\
\text { direction) }\end{array}$ \\
\hline$\Gamma$ & Modal damping matrix $[1 / \mathrm{s}]$ & $F_{c}$ & Tool tip force, generic direction $[\mathrm{N}]$ \\
\hline$\Omega^{2}$ & Modal Stiffness matrix $\left[\mathrm{rad}^{2} / \mathrm{s}^{2}\right]$ & $A, B, C, D$ & State space matrices \\
\hline$F$ & Vector of model input force $[\mathrm{N}]$ & $\boldsymbol{G}$ & System noise matrix \\
\hline$g$ & Output shape matrix & $Z$ & Measurement noise \\
\hline$q$ & Vector of modal coordinates $[\mathrm{m}]$ & $w$ & System noise \\
\hline$x$ & System model states & $\boldsymbol{K}$ & Kalman filter gain matrix \\
\hline$u$ & System model inputs & $\boldsymbol{P}$ & State estimation error covariance \\
\hline$p$ & Vector of model coordinates $[\mathrm{m}]$ & $\boldsymbol{R}$ & Measurement noise matrix covariance \\
\hline$y$ & $\begin{array}{l}\text { Vector of system outputs, } \\
\text { displacement }[\mathrm{m}]\end{array}$ & $Q$ & System noise matrix covariance \\
\hline$v$ & $\begin{array}{l}\text { Velocity vector }[\mathrm{m} / \mathrm{s}] \\
\text { of the system outputs }\end{array}$ & $x_{e}$ & Vector of system states \\
\hline$a$ & $\begin{array}{l}\text { Acceleration vector }\left[\mathrm{m} / \mathrm{s}^{2}\right] \\
\text { of the system outputs }\end{array}$ & $\hat{x}_{e}$ & Estimated vector of system states \\
\hline$y_{n}$ & $\begin{array}{l}\text { Vector of system outputs } \\
\text { (displacements and accelerations) }\end{array}$ & $\theta$ & Tool tip bending coordinate [rad] \\
\hline$y_{e}$ & Vector of system expanded outputs & $\hat{\gamma}_{l m}(f)$ & Coherence function estimation \\
\hline$n s$ & Number of model outputs & $\widehat{H}_{l m}(f)$ & Cross-spectrum estimation \\
\hline$n$ & Number of identified eigenmodes & $\widehat{H}_{l l}(f), \widehat{H}_{m m}(f)$, & Auto-spectra estimation \\
\hline$\phi$ & Eigenvector matrix & $c$ & Covariance matrix \\
\hline$\omega_{j}$ & Pole frequency $[\mathrm{rad} / \mathrm{s}]$ & $\boldsymbol{r}_{l m}(l, m)$ & Correlation coefficient \\
\hline$\zeta_{j}$ & Damping ratio & $n_{d}$ & $\begin{array}{l}\text { number of signal records used for the } \\
\text { coherence function computation }\end{array}$ \\
\hline
\end{tabular}

Chae et al. [14] measured the micro cutting forces using a three-axis miniature force sensor and accelerometers. They utilized Kalman filter in order to extend the bandwidth of the force measurement system, compensate for the structural dynamics and fuse the sensor signals information. Salehi et al [15] extended the previous research results using a sensor fusion approach but only preliminary results were achieved. Möhring et al. [16] developed a sensing fixture (equipped with different sensors) and an adaptive sensorized spindle in order to monitor both the process (cutting forces) and the machine conditions and to compensate for workpiece static deflections. With the help of a sensor fusion approach, they extended the bandwidth of the forces measurements. Denkena and Hackelöer [17] estimated disturbance force of dynamic mechanical systems using Kalman filtering techniques. It was also reported that multiple sensors usage can improve the estimation accuracy and model inaccuracies. The approach was verified on a milling machine with active magnetic guides. Nevertheless, the above-mentioned works are limited to the laboratory scales due to the complexity of the experimental setups that cannot be easily transferred to industrial scales.

Since machined surface finish is increasingly being concerned in industrial products, much attention has to be paid to the study of the effects of cutting vibration on surface quality. So far, research has dealt with two main approaches: the first one is based on cutting process simulation models while the second exploits vibrational measurements [18]. Referring to the first approach, many researchers, e.g. Montgomery and Altintas [19], Paris et al. [20] and Peigne et al. [21], developed dynamic models of the machining processes to simulate the surface profile generation. However, these models are far to be able to predict the exact cutting vibrations because most of them are greatly simplified. Hence, in order to guarantee a more reliable surface quality estimation, cutting vibration measurements should feed a proper surface 
generating model. Indeed, Lee et al. [22] studied the effects of cutting vibrations on surface finish in turning by real-time measuring the tool-workpiece relative displacements. However, the on-line measurement of tool-part relative vibration is a particularly difficult task in milling operations. Jiang et al. [18] developed a model based methodology for estimating the machined surface on the base of vibration measurements. In order to perform such measurements, they used eddy current displacement sensors placed close to the tool tip and to the workpiece. Nonetheless, because of the sensors location and the setup configuration, the methodology is limited to the experimental and laboratory scales.

In order to overcome the main analyzed limitations, in this paper, an in-process model based observer of cutting forces and tool tip vibration was designed and tested on a machine center. The observer relies on signals coming from a triaxial accelerometer mounted in the spindle housing and on inner inductive sensors that measure the relative spindle housingshaft radial displacements. The observer was based on Kalman filtering theory and compensates, for both forces and vibrations, the effects of the machine structure low frequency dynamics and the spindle high frequency dynamics. The observer, fusing the data coming from the sensors, assures the extension of the frequency bandwidth of the performed estimations. In this manner, the developed system can be used over a vast range of machining operations. The observer is based on the machine tool dynamic model that was identified by means of an experimental modal analysis. A final experimental campaign was performed in order to analyze the accuracy of the estimations. Specific tests were arranged to evaluate the extension of the estimations frequency bandwidth. The observer was also tested during milling tests.

This research has distinct contributions as compared to the analyzed literature papers. A multi-sensors model based observer was developed in order to estimate, at the same time, both the cutting forces and the tool tip vibrations. This information is very useful for process and part quality monitoring. The proposed approach, compensating the machine tool dynamics over a wide frequency range, makes it applicable to different milling operations. To the author knowledge, no other literature works present such contents. Moreover, the developed observer relies on sensors that can be easily integrated, as demonstrated in this work, in a commercial electrospindle thus assuring an easy industrial diffusion.

The paper is organized as follows. In section 2 the modelling procedure is described, in section 3 the observer development is explained. The results of the final experimental sessions are reported and critically discussed in section 4.

\section{Machine tool modelling and experimental characterization}

\subsection{Experimental setup and system model identification}

The objective of this work is to estimate the force applied to the tool, tool tip vibration and tool bending deflection in milling applications, only relying on signals that can be available on industrial machine tools. In order to achieve this goal, a suitable model-based observer based on Kalman filtering is developed. The observer is designed in order to be fed by the spindle housing accelerations and the spindle shaft-housing relative displacements, Fig. 2 .

The observer is based on the machine tool dynamic model, further analytical details on the developed model are reported in section 2.2. The model parameters were obtained performing an experimental modal analysis on the machine. The machine was excited with force pulses applied at tip of the tool. Different sensorized hammers were used to excite the machine dynamics over a wide frequency range (up to $1500 \mathrm{~Hz}$ ), Table 1. For both $\mathrm{X}$ and $\mathrm{Y}$ machine directions, the following quantities were defined: tool tip force (excitation input, $\mathrm{F}_{\mathrm{c}}$ ), tool vibration at point 1 and at point 2, spindle housing vibration and the relative radial displacement between spindle shaft and spindle housing. Moreover, vibrations revealed at point 1 and point 2 can be used for computing the tool tip bending deflection. The abovementioned quantities represent the degrees of freedom of the machine tool dynamic model. The whole experimental characterization procedure is summarized in Fig. 1. The experimental set-up illustrated in Fig. 2 was not only used in the model characterization phase but it was also exploited for some of the final qualifying tests. Through a schematic representation in the Y-Z plane, Fig. 3 also shows the role of each sensor in different performed experimental phases. The sensors map depicted in Fig. 3 was also adopted for the X-Z plane. The accelerometers placed on the tool tip (point 1 and point 2) were used in the characterization phase. The inductive relative displacement sensors placed in correspondence of the same points were used during some of the final experimental tests in order to analyze the observer capability of estimating the vibration of the tool. As can be noted in Fig. 2, the additional inductive relative displacement sensors were positioned close to the tool by means of an external slender holding structure. The absence of any dynamic coupling between the external structure and the machine was verified during the tests using another additional accelerometer (labelled as point 5). This mainly guaranteed the reliability of the ground-relative tool vibration measurements performed with the external inductive sensors. During the final cutting tests, only the inductive sensors located at point 2 (X and Y directions) were used. For sake of clarity, a list of the used sensors is reported in the Table 1. 


\section{Machine Tool Modelling Process}

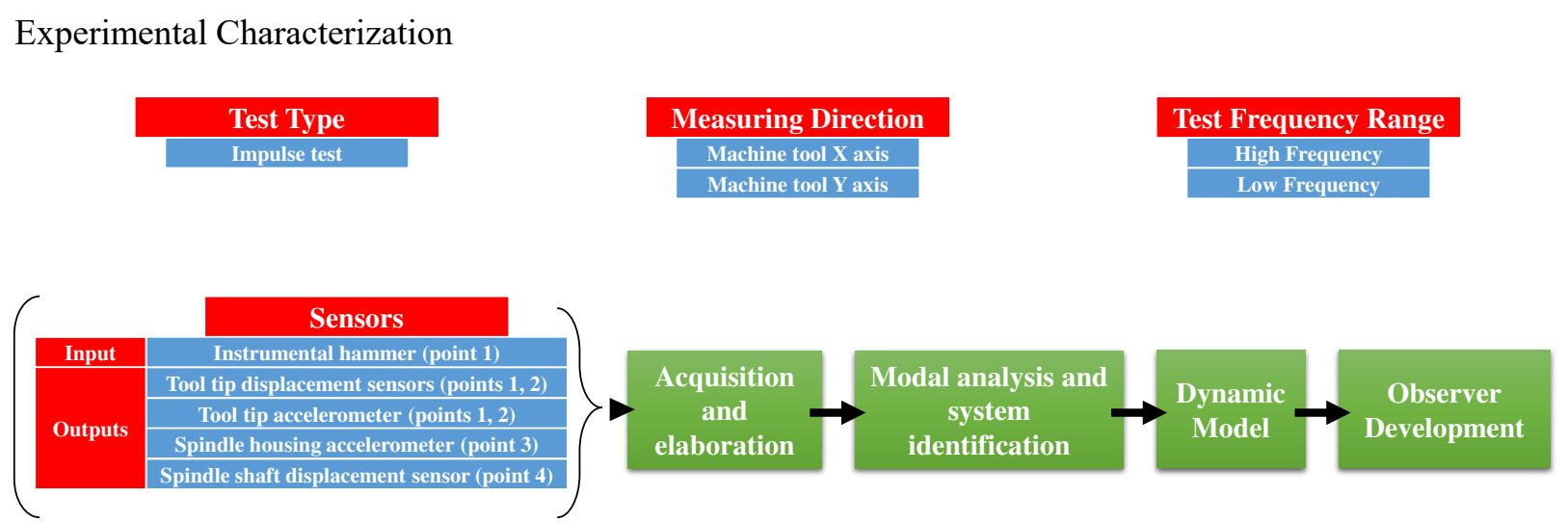

Fig. 1. Machine tool model identification - procedure overview.
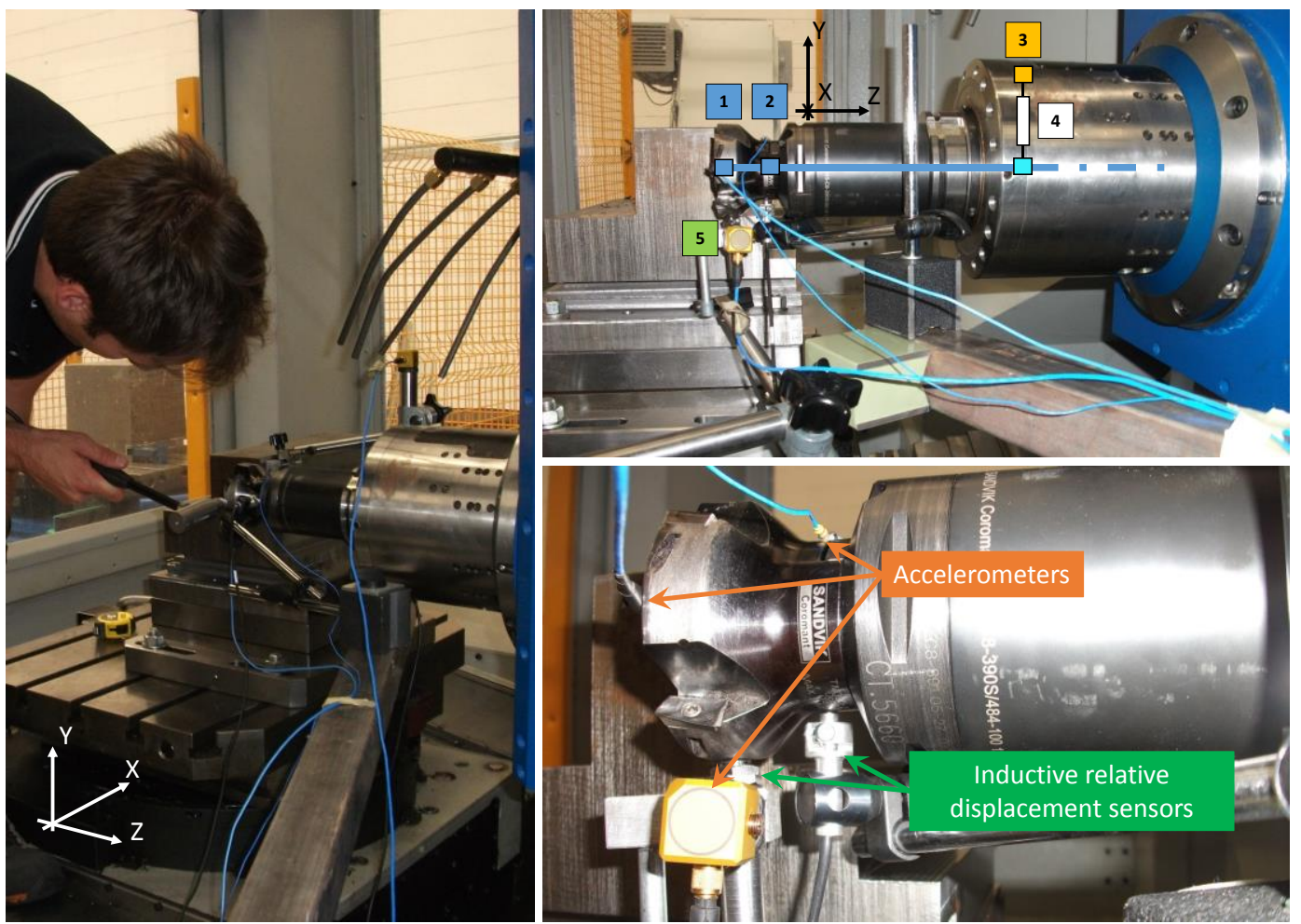

Fig. 2. X direction measurement (left), sensors positions map (right above) and tool tip area (right below).

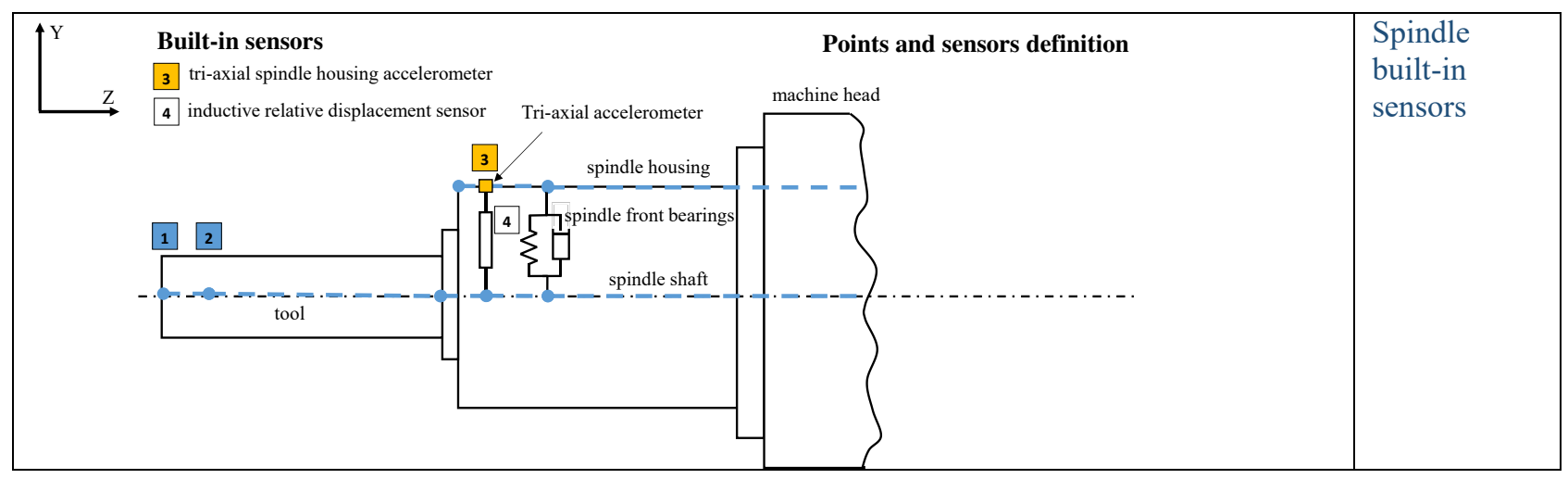




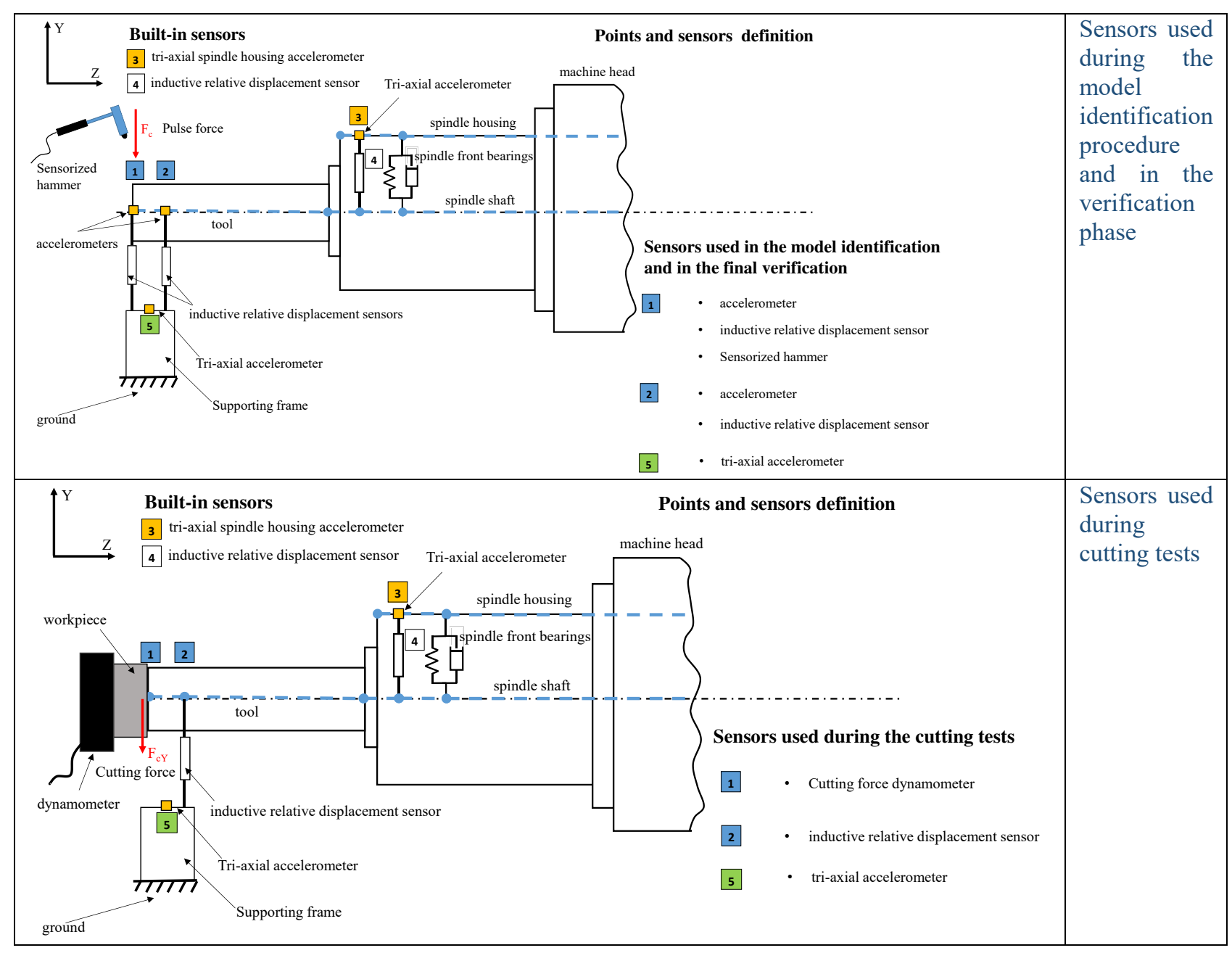

Fig. 3: schematic representation of the sensors used during different experimental phases

Some of the measured frequency response functions (FRFs) are reported in Fig. 4, both for X and Y directions. In particular, dynamic compliances (displacement/force) at the tool tip (point 1, point 2), at the spindle housing (point 3) and the FRFs performed using the inner relative displacements sensors (point 4) are shown. In addition, as an example of the performed modal analysis, the identified FRFs (point 1) are also depicted in Fig. 4. The list of the identified eigenmodes is reported in Table 2. Looking at Fig. 4 it can be observed that the identified modal model accurately reproduces the experimental tests. The bending displacements of the tool tip were calculated and the relative FRFs in both $\mathrm{X}$ and $\mathrm{Y}$ directions are reported in Fig. 5. The bending coordinates are calculated with a linear approach by computing the angle from the displacement quantities registered at point 1 and point 2. As already demonstrated in [18], both the radial tool tip displacements and the tool bending deflections are important information that could feed a model that estimates the quality of the processed parts. The approach developed in this paper could simplify the experimental set up used in [18]. As can be observed in Fig. 4 and Fig. 5, the low frequency eigenmodes are mainly related to the machine structure (i.e. comprising the spindle headstock), see also [23]. The medium-high frequency eigenmodes are mainly related to the spindle-tool dynamics. It can be observed that measurements performed at the tool tip (both points 1 and 2) exhibit eigenmodes over the whole frequency range whereas the spindle housing compliance (point 3) contains only low-medium frequency dynamics. Since the dynamic compliance at point 4 describes mainly the relative spindle shaft-housing dynamics, no eigenmodes can be appreciated in the low-medium frequency range. Indeed, in this frequency range, the relative dynamic compliance exhibits only a static stiffness-like behavior. A similar behavior can be observed for the FRFs reported in Fig. 5. For this reason, in the current work, an observer that exploits data coming not only from a single sensor (as done in [9]) has been developed. The introduced enhancement allows extending the observer compensating properties and makes it suitable for different milling scenarios. 


\begin{tabular}{|c|c|c|c|c|}
\hline position & measurement & sensor type & specification & Sensitivity \\
\hline \multirow[t]{2}{*}{1} & tool tip vibration & accelerometer & РCB 356A32 & $100 \mathrm{mV} / \mathrm{g}$ \\
\hline & tool tip vibration & $\begin{array}{l}\text { inductive relative displacement } \\
\text { sensor }\end{array}$ & KEYENCE EX 110V & $0.01 \mathrm{~V} / \mathrm{mm}$ \\
\hline \multirow[t]{2}{*}{2} & tool tip vibration & accelerometer & PCB 352A24 & $100 \mathrm{mV} / \mathrm{g}$ \\
\hline & tool tip vibration & $\begin{array}{l}\text { inductive relative displacement } \\
\text { sensor }\end{array}$ & KEYENCE EX 305V & $0.01 \mathrm{~V} / \mathrm{mm}$ \\
\hline 3 & spindle housing vibration & accelerometer & DYTRAN 3263A2 & $100 \mathrm{mV} / \mathrm{g}$ \\
\hline 4 & $\begin{array}{l}\text { spindle shaft-housing relative } \\
\text { displacement }\end{array}$ & $\begin{array}{l}\text { inductive relative displacement } \\
\text { sensor }\end{array}$ & KEYENCE EX 305V & $0.01 \mathrm{~V} / \mathrm{mm}$ \\
\hline 5 & external structure vibration & accelerometer & PCB 356B18 & $1000 \mathrm{mV} / \mathrm{g}$ \\
\hline 1 & tool tip force & $\begin{array}{l}\text { instrumented hammer (low } \\
\text { freq.) }\end{array}$ & PCB 086D20 (rubber tip) & $0.23 \mathrm{mV} / \mathrm{N}$ \\
\hline 1 & tool tip force & $\begin{array}{l}\text { instrumented hammer (high } \\
\text { freq.) }\end{array}$ & PCB 086D05 (nylon tip) & $0.23 \mathrm{mV} / \mathrm{N}$ \\
\hline
\end{tabular}
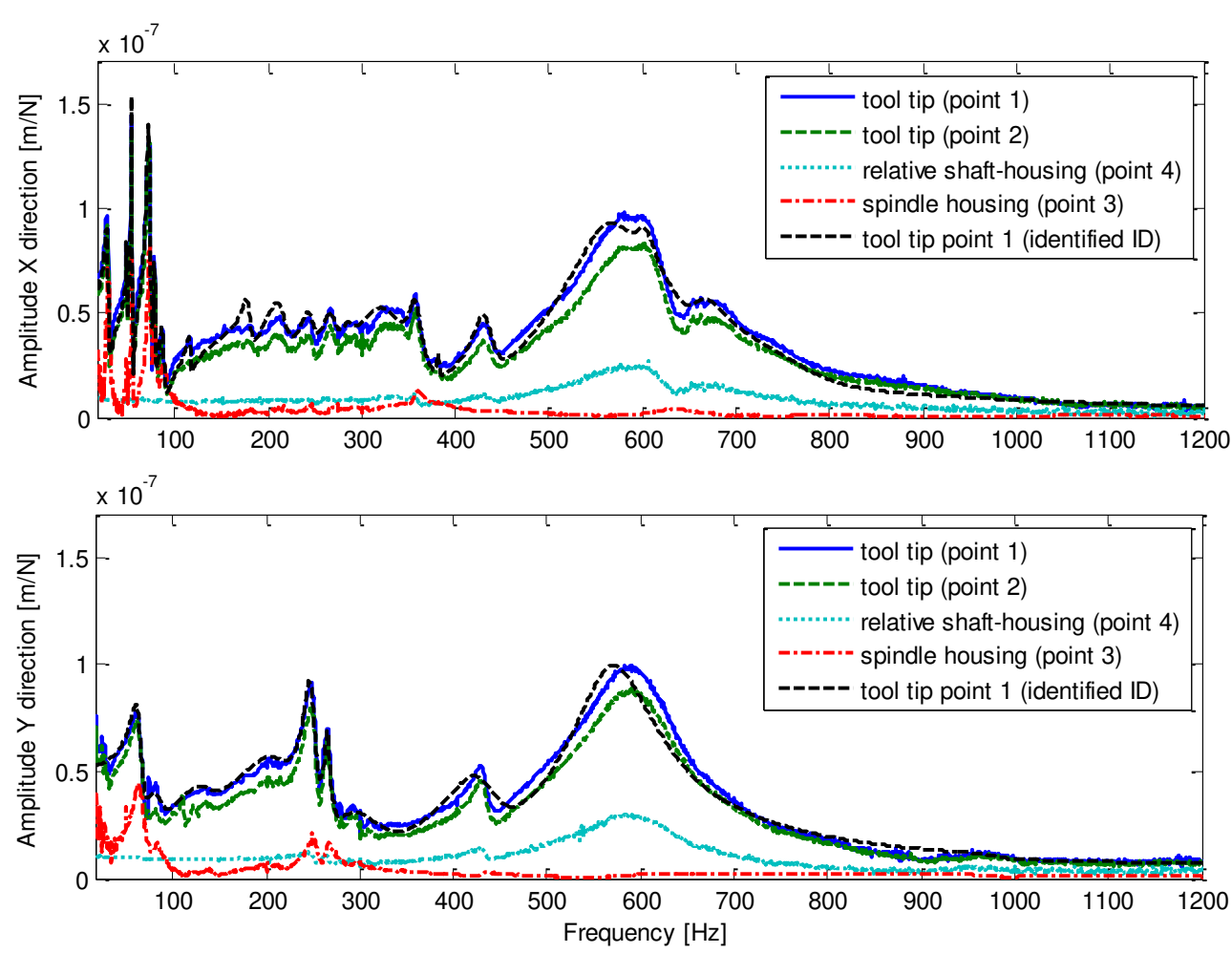

Fig. 4. Experimental FRFs (amplitudes) along X-Y directions and the fitted tool tip dynamic compliances (identified ID).

Table 2. Identified eigenmodes for $\mathrm{X}$ and $\mathrm{Y}$ directions

\begin{tabular}{ccccccccc}
\hline \multicolumn{9}{c}{ X direction } \\
Mode & $\omega_{j} /(2 \pi)[\mathrm{Hz}]$ & $\zeta_{j}$ & Mode & $\omega_{j} /(2 \pi)[\mathrm{Hz}]$ & $\zeta_{j}$ & Mode & $\omega_{j} /(2 \pi)[\mathrm{Hz}]$ & $\zeta_{j}$ \\
\hline 1 & 28.0 & 0.069 & 12 & 288.0 & 0.033 & 1 & 64.2 & 0.096 \\
2 & 48.8 & 0.006 & 13 & 329.7 & 0.097 & 2 & 84.7 & 0.142 \\
3 & 54.2 & 0.008 & 14 & 357.8 & 0.019 & 3 & 138.0 & 0.131 \\
4 & 72.6 & 0.027 & 15 & 381.9 & 0.003 & 4 & 211.1 & 0.129 \\
5 & 78.5 & 0.013 & 16 & 433.0 & 0.025 & 5 & 247.6 & 0.030 \\
6 & 86.5 & 0.035 & 17 & 568.2 & 0.088 & 6 & 265.6 & 0.012 \\
7 & 116.2 & 0.015 & 18 & 600.8 & 0.018 & 7 & 299.1 & 0.100 \\
8 & 179.7 & 0.025 & 19 & 662.9 & 0.028 & 8 & 430.6 & 0.088 \\
9 & 214.9 & 0.054 & 20 & 769.6 & 0.087 & 9 & 571.4 & 0.077
\end{tabular}




\begin{tabular}{|c|c|c|c|c|c|c|c|c|}
\hline 10 & 245.8 & 0.032 & 21 & 1288.0 & 0.023 & 10 & 960.7 & 0.031 \\
\hline 11 & 268.9 & 0.031 & & & & 11 & 1214.0 & 0.026 \\
\hline
\end{tabular}

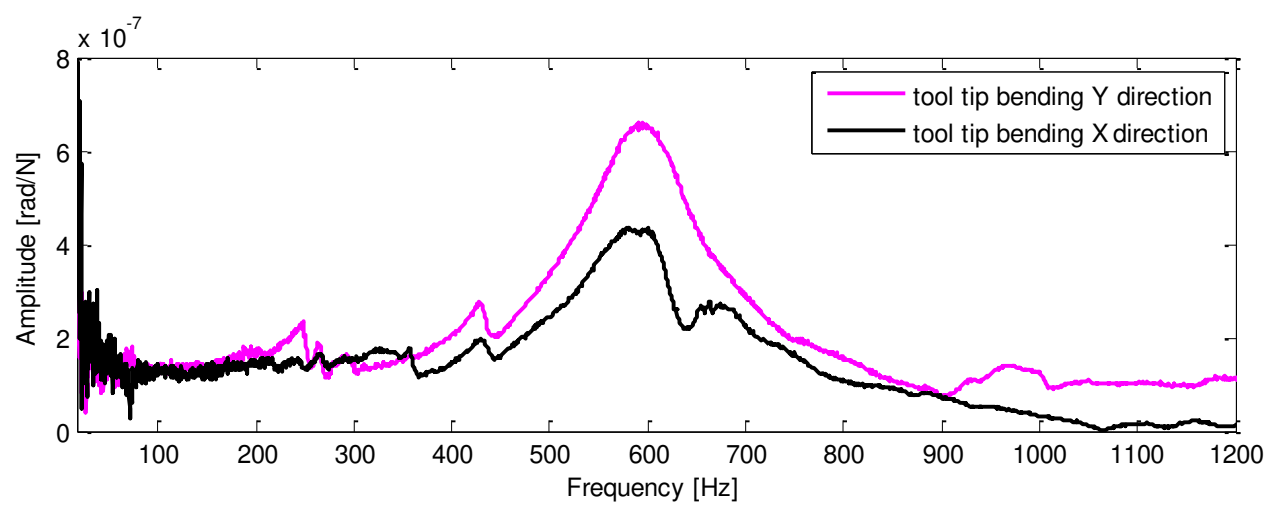

Fig. 5. Experimental FRFs (amplitudes) of tool tip bending coordinates along X and Y directions.

\subsection{Machine tool dynamic modelling}

The model developed through the experimental modal analysis is described by Eq. (1) and Eq. (2). Eq. (1) describes the system dynamics, e.g. the relationship between the model inputs $F(s)$ and the model coordinates $p(s)$. Eq. (2) shows the relationship between the model coordinates $p(s)$ and the system output vector $y(s)$ that is the vector that contains the quantities measured by the sensors.

$$
\begin{aligned}
& {\left[\boldsymbol{M} s^{2}+\boldsymbol{R}_{d} s+\boldsymbol{K}_{s}\right]_{(n \times n)} p(s)_{(n \times 1)}=F(s)_{(n \times 1)}} \\
& y(s)_{(n s \times 1)}=\boldsymbol{g}_{(n s \times n)} p(s)_{(n \times 1)}
\end{aligned}
$$

$F(s)$ is a vector containing the input forces applied to the system. $\boldsymbol{M}, \boldsymbol{R}_{d}, \boldsymbol{K}_{s}$ are mass, damping and stiffness matrices respectively and $\boldsymbol{g}$ is the output shape matrix. Furthermore, $n$ is the number of eigenmodes considered in the modal identification of the system and $n s$ is the number of outputs of the measurement vector. In this case the input force is applied at the tool tip (point 1) in X and Y directions. The other elements of the vector are null.

It is possible to get the system modal coordinate $q(s)$ by means of a modal transformation performed on the system coordinates, Eq. (3).

$$
p(s)_{(n \times 1)}=\boldsymbol{\Phi}_{(n \times n)} q(s)_{(n \times 1)}
$$

Where $\boldsymbol{\Phi}$ is the eigenvectors matrix. Eq. (1) and Eq. (2) are then substituted by Eq. (4) and Eq. (5), as follows:

$$
\begin{aligned}
& {\left[\boldsymbol{I}^{2}+\boldsymbol{\Gamma} s+\boldsymbol{\Omega}^{2}\right] q(s)=\boldsymbol{\Phi}^{T} F(s)} \\
& y(s)=\boldsymbol{g} \boldsymbol{\Phi} q(s)=\boldsymbol{C}^{\prime} q(s)
\end{aligned}
$$

$\boldsymbol{\Gamma}$ and $\boldsymbol{\Omega}^{2}$ are expressed as follows:

$$
\boldsymbol{\Gamma}=\left[\begin{array}{ccccc}
\Gamma_{1} & 0 & 0 & 0 & 0 \\
0 & \ddots & 0 & 0 & 0 \\
0 & 0 & \Gamma_{j} & 0 & 0 \\
0 & 0 & 0 & \ddots & 0 \\
0 & 0 & 0 & 0 & \Gamma_{n}
\end{array}\right]=\left[\begin{array}{ccccc}
2 \zeta_{1} \omega_{1} & 0 & 0 & 0 & 0 \\
0 & \ddots & 0 & 0 & 0 \\
0 & 0 & 2 \zeta_{j} \omega_{j} & 0 & 0 \\
0 & 0 & 0 & \ddots & 0 \\
0 & 0 & 0 & 0 & 2 \zeta_{n} \omega_{n}
\end{array}\right] ; \quad \mathbf{\Omega}^{2}=\left[\begin{array}{ccccc}
\omega_{1}^{2} & 0 & 0 & 0 & 0 \\
0 & \ddots & 0 & 0 & 0 \\
0 & 0 & \omega_{j}^{2} & 0 & 0 \\
0 & 0 & 0 & \ddots & 0 \\
0 & 0 & 0 & 0 & \omega_{n}^{2}
\end{array}\right]
$$

where $\omega_{j}^{2}$ is linked to the eigenvalue $j$ and $\zeta_{j}$ is the corresponding damping ratio. Each dynamic compliance $\alpha(\mathrm{s}){ }_{j, k}$ (model output $j$, model input $k$ ) can be expressed using Eq. (7) where the contribution of each single identified normal eigenmode is clearly visible.

$$
\frac{y}{F}(s)_{j, k}=\alpha(s)_{j, k}=\sum_{r=1}^{n} \frac{g_{j, r} \phi_{j, r} \phi_{k, r}}{s^{2}+2 \zeta_{j} \omega_{j} s+\omega_{j}^{2}}
$$


In our specific case, the identified eigenmodes, both for $\mathrm{X}$ and $\mathrm{Y}$ directions, are reported in Table 2. In order to proceed with the design of the Kalman filter, the system model was converted into state-space representation as in Eq. (8), where $\boldsymbol{A}$ describes the system dynamics, $\boldsymbol{B}$ is the input matrix, $\boldsymbol{C}$ is the output matrix, $\boldsymbol{D}$ is the feed forward matrix. $u$ is the input vector and $y$ is the output vector.

$$
\left\{\begin{array}{l}
\dot{x}_{(2 n \times 1)}=\boldsymbol{A}_{(2 n \times 2 n)} x_{(2 n \times 1)}+\boldsymbol{B}_{(2 n \times n)} u_{(n \times 1)} \\
y_{(n s \times 1)}=\boldsymbol{C}_{(n s \times 2 n)} x_{(2 n \times 1)}+\boldsymbol{D}_{(n s \times n)} u_{(n \times 1)}
\end{array}\right.
$$

In this case, the system outputs are:

$$
y=\left\{\begin{array}{c}
y_{1} \\
y_{2} \\
y_{3} \\
y_{r e l} \\
\theta
\end{array}\right\}=\left\{\begin{array}{l}
\text { tool vibration (displacement point 1) } \\
\text { tool vibration (displacement point 2) } \\
\text { housing vibration (displacement point 3) } \\
\text { relative displacement (point 4) } \\
\text { tool tip bending coordinate }
\end{array}\right\}
$$

In the following equations, $x$ is introduced as the system states vector and $u$ is the vector of the system inputs (as reported in Eq. (10)).

$$
x=\left\{\begin{array}{llllllll}
q_{1} & \dot{q}_{1} & q_{2} & \dot{q}_{2} & \cdots & \cdots & q_{n} & \dot{q}_{n}
\end{array}\right\}^{T} ; \quad u=F(s)
$$

The matrices of the state space representation can be computed as shown in Eq. (11) and Eq. (12).

$$
\begin{aligned}
\boldsymbol{A} & =\left[\begin{array}{ccccccc}
0 & 1 & \vdots & \vdots & \vdots & \vdots & 0 \\
-\omega_{1}^{2} & -\Gamma_{1} & \vdots & \vdots & \vdots & 0 & 0 \\
0 & 0 & 0 & 1 & \vdots & \vdots & 0 \\
0 & 0 & -\omega_{2}^{2} & -\Gamma_{2} & \vdots & 0 & 0 \\
\vdots & \vdots & \vdots & \vdots & \vdots & \vdots & \vdots \\
\vdots & \vdots & \vdots & \vdots & \vdots & \vdots & \vdots \\
0 & 0 & \vdots & \vdots & \vdots & 0 & 1 \\
0 & 0 & \vdots & \vdots & \vdots & -\omega_{n}^{2} & -\Gamma_{n}
\end{array}\right] ; \quad \boldsymbol{B}=\left[\begin{array}{cccc}
0 & & 0 \\
\phi_{1,1}^{T} & \vdots & \phi_{1, n}^{T} \\
0 & \vdots & 0 \\
\phi_{2,1}^{T} & \vdots & \phi_{2, n}^{T} \\
\vdots & \vdots & \vdots \\
\vdots & \vdots & \vdots \\
0 & \vdots & 0 \\
\phi_{n, 1}^{T} & \vdots & \phi_{n, n}^{T}
\end{array}\right] \\
\boldsymbol{C} & =\left[\begin{array}{ccccccccc}
c_{1,1}^{\prime} & 0 & c_{1,2}^{\prime} & \cdots & c_{1, n}^{\prime} & 0 \\
c_{2,1}^{\prime} & 0 & c_{2,2}^{\prime} & \cdots & c_{2, n}^{\prime} & 0 \\
\vdots & \vdots & \vdots & \ddots & \vdots & \vdots \\
c_{i, 1}^{\prime} & 0 & c_{i, 2}^{\prime} & \cdots & c_{i, n}^{\prime} & 0 \\
\vdots & \vdots & \vdots & \ddots & \vdots & \vdots \\
c_{n s, 1}^{\prime} & 0 & c_{n s, 2}^{\prime} & \cdots & c_{n s, n}^{\prime} & 0
\end{array}\right]=\left[\begin{array}{cccccc}
c_{1,1} & c_{1,2} & c_{1,3} & \cdots & c_{1,2 n-1} & c_{1,2 n} \\
c_{2,1} & c_{2,2} & c_{2,3} & \cdots & c_{2,2 n-1} & c_{2,2 n} \\
\vdots & \vdots & \vdots & \ddots & \vdots & \vdots \\
c_{i, 1} & c_{i, 2} & c_{i, 3} & \cdots & c_{i, 2 n-1} & c_{i, 2 n} \\
\vdots & \vdots & \vdots & \ddots & \vdots & \vdots \\
c_{n s, 1} & c_{n s, 2} & c_{n s, 3} & \cdots & c_{n s, 2 n-1} & c_{n s, 2 n}
\end{array}\right] ; \quad \boldsymbol{D}=[\mathbf{0}]
\end{aligned}
$$

In order to feed the Kalman-based observer directly with the sensors data (e.g. the spindle shaft-housing relative displacements and the housing accelerations) the developed model is modified as follows. Since the system output matrix $y$ is expressed as a linear combination of the system states $q$ through the elements of matrix $\boldsymbol{C}$, the corresponding vector of velocities $v$ can be expressed by Eq. (13).

$$
v=\dot{y}=\left[\begin{array}{ccccccc}
0 & c_{1,1} & 0 & c_{1,3} & 0 & \vdots & c_{1,2 n-1} \\
0 & c_{2,1} & 0 & c_{2,3} & 0 & \vdots & c_{2,2 n-1} \\
\vdots & \vdots & \vdots & \vdots & \vdots & \vdots & \vdots \\
0 & c_{i, 1} & 0 & c_{i, 3} & \vdots & \vdots & c_{i, 2 n-1} \\
\vdots & \vdots & \vdots & \vdots & \vdots & \vdots & \vdots \\
0 & c_{n s, 1} & 0 & c_{n s, 3} & 0 & \vdots & c_{n s, 2 n-1}
\end{array}\right] x=C_{v e l(n s \times 2 n)} x_{(2 n \times 1)}
$$

The same approach is also adopted to compute the system model output accelerations, according to the Eq. (14).

$$
a=\dot{v}=\left[\begin{array}{ccccccc}
0 & c_{1,1} & 0 & c_{1,3} & 0 & \vdots & c_{1,2 n-1} \\
0 & c_{2,1} & 0 & c_{2,3} & 0 & \vdots & c_{2,2 n-1} \\
\vdots & \vdots & \vdots & \vdots & \vdots & \vdots & \vdots \\
0 & c_{i, 1} & 0 & c_{i, 3} & \vdots & \vdots & c_{i, 2 n-1} \\
\vdots & \vdots & \vdots & \vdots & \vdots & \vdots & \vdots \\
0 & c_{n s, 1} & 0 & c_{n s, 3} & 0 & \vdots & c_{n s, 2 n-1}
\end{array}\right] \dot{x}=C_{v e l(n s \times 2 n)} \dot{x}_{(2 n \times 1)}
$$


The derivative of the system states is computed taking into account the state equation in Eq. (8), this leads to Eq. (15).

$$
a=\boldsymbol{C}_{v e l}(\boldsymbol{A} x+\boldsymbol{B} u)=\boldsymbol{C}_{a c c} x+\boldsymbol{D}_{a c c} u
$$

In order to add the accelerations as additional outputs, it is necessary to arrange the state-space system representation as follows (see Eq. (16)).

$$
\left\{\begin{array}{l}
\dot{x}=\boldsymbol{A} x+\boldsymbol{B} u \\
\left\{\begin{array}{l}
y \\
a
\end{array}\right\}_{(2 n s \times 1)}=\left[\begin{array}{l}
\boldsymbol{C}_{(n s \times 2 n)} \\
\boldsymbol{C}_{a c c(n s \times 2 n)}
\end{array}\right] x_{(2 n \times 1)}+\left[\begin{array}{l}
\boldsymbol{D}_{(n s \times n)} \\
\boldsymbol{D}_{a c c(n s \times n)}
\end{array}\right] u_{(n \times 1)}=\boldsymbol{C}_{e x t} x+\boldsymbol{D}_{\text {ext }} u
\end{array}\right.
$$

In this case, only the generic acceleration $a_{i}$ is added to the output thus, the final representation of the system can be outlined in Eq. (17).

$$
\left\{\begin{array}{l}
\dot{x}=\boldsymbol{A} x+\boldsymbol{B} u \\
y_{n}=\left\{\begin{array}{c}
y \\
a_{i}
\end{array}\right\}_{((n s+1) \times 1)}=\boldsymbol{C}_{n_{((n s+1) \times 2 n)}} x+\boldsymbol{D}_{n_{((n s+1) \times n)}} u
\end{array}\right.
$$

The same machine model described by Eq. (17) has been developed both for X and Y axes. In this context, the considered numbers of identified eigenmodes along with output vector dimension are reported as follows.

- $n_{x}=21$

- $n_{y}=11$

- $n s=5$

$\mathrm{n}_{\mathrm{x}}$ and $\mathrm{n}_{\mathrm{y}}$ depend on the dynamics of the considered machine tools and on the identification procedure. In some cases, even the change of the tool can significantly modify the machine dynamics especially in the high frequency range hence, a new experimental modal analysis and the above described procedure need to be performed again.

Before developing the Kalman-based observer, the observability of the model was investigated by checking the observability matrix to be full rank according to the research work reported in [9].

\section{Observer design}

A Kalman-based observer is an optimal model-based state estimator that is able to estimation the state vector $\hat{x}$ as well as some other non-easily measurable quantities (model outputs $y$ ) exploiting real-time data coming from the plant (machine).

In this section the created machine dynamic model is re-arranged in order to be used as an observer that indirectly estimates the tool tip applied force $F_{c}$, the tool tip displacements $\left(y_{1}, y_{2}\right)$ and the tool bending deflection $\theta$. These quantities are typically very difficult to be measured during a real milling operation. Since we are interested into estimating the force applied to the tool, it has to be expanded to the state vector as an additional observer state, Eq. (18).

$$
\left\{\begin{array}{lllllllll}
q_{1} & \dot{q}_{1} & q_{2} & \dot{q}_{2} & \cdots & \cdots & q_{n} & \dot{q}_{n} & F_{c}
\end{array}\right\}^{T}=x_{e_{((2 n+1) \times 1)}}
$$

For each single machine axes direction (X or Y), the re-arranging procedure leads to Eq. (19). As can be easily noted, the observer relies on spindle housing-shaft relative displacement $y_{r e l}$ and the spindle housing acceleration $a_{s h}$.

$$
\left\{\begin{array}{l}
\dot{x}_{e}=\boldsymbol{A}_{e} x_{e}+\boldsymbol{G}_{(2 n \times 1)} w_{(1 \times 1)} \\
y_{e}=\left\{\begin{array}{l}
y_{r e l} \\
a_{s h}
\end{array}\right\}_{(2 \times 1)}=\boldsymbol{C}_{\mathrm{e}} x_{e}+z_{(2 \times 1)}
\end{array}\right.
$$

The matrix that describes the dynamics of the expanded model is defined according to Eq. (20), 


$$
\boldsymbol{A}_{e}=\left[\begin{array}{ccc}
\boldsymbol{A} & \boldsymbol{B}\left\{\begin{array}{c}
1 \\
0 \\
\vdots \\
\vdots \\
0
\end{array}\right\}_{(n \times 1)} \\
& & \\
{\left[\begin{array}{lll}
0 & 0 & \cdots
\end{array}\right]_{(1 \times 2 n)}} & 0
\end{array}\right]_{((2 n+1) \times(2 n+1))}
$$

In addition, the new output matrix is defined in Eq. (21) and Eq. (22),

$$
\boldsymbol{C}_{e}=\left[\begin{array}{ll}
\boldsymbol{C}_{n 1} & \boldsymbol{D}_{n 1}
\end{array}\right]_{(2 \times(2 n+1))}
$$

where

$$
\boldsymbol{D}_{n 1}=\left[\begin{array}{c}
D_{n_{4,1}} \\
D_{n_{6,1}}
\end{array}\right]_{(2 \times 1)} ; \quad \boldsymbol{C}_{n 1}=\left[\begin{array}{lll}
C_{n_{4,1}} & \cdots & C_{n_{4,2 n}} \\
C_{n_{6,1}} & \cdots & C_{n_{6,2 n}}
\end{array}\right]_{(2 \times 2 n)}
$$

The matrix $\boldsymbol{G}$ expressed in Eq. (19) is the system noise matrix, $w$ is the zero-mean white Gaussian process noise, $z$ is zeromean white Gaussian measurement noise, and therefore, $\boldsymbol{Q}=E\left[w w^{T}\right]>0$ and $\boldsymbol{R}=E\left[z z^{T}\right]>0$ are their covariance matrices, respectively.

\subsection{Observer tuning}

A Kalman-based observer can minimize state estimation errors, $\tilde{x}=\hat{x}-x$. The Kalman gain matrix $\boldsymbol{K}$ (Eq. (24)) is determined by minimization of the error covariance matrix $\boldsymbol{P}=E\left[\begin{array}{ll}\tilde{x} & \tilde{x}^{T}\end{array}\right]$. The minimum error covariance matrix $\boldsymbol{P}$ can be obtained by solving the Riccati equation as follows [9],

$$
\begin{aligned}
& \dot{\boldsymbol{P}}=\boldsymbol{A}_{e} \boldsymbol{P}+\boldsymbol{P} \boldsymbol{A}_{e}{ }^{T}+\boldsymbol{Q}-\boldsymbol{P} \boldsymbol{C}_{e}{ }^{T} \boldsymbol{R}^{-1} \boldsymbol{C}_{e} \boldsymbol{P} \\
& \boldsymbol{K}=\boldsymbol{P} \boldsymbol{C}_{e}{ }^{T} \boldsymbol{R}^{-1}
\end{aligned}
$$

The system state estimation can be performed using the following equation,

$$
\dot{\hat{x}}_{e}=\boldsymbol{A}_{e} \hat{x}_{e}+\boldsymbol{K}\left(y_{e}-\hat{y}_{e}\right)=\boldsymbol{A}_{e} \hat{x}_{e}+\boldsymbol{K}\left(y_{e}-\boldsymbol{C}_{e} \hat{x}_{e}\right)=\left(\boldsymbol{A}_{e}-\boldsymbol{K} \boldsymbol{C}_{e}\right) \hat{x}_{e}+\boldsymbol{K} y_{e}
$$

The estimation of the desired system outputs can be performed through Eq. (26),

$$
\hat{y}_{0}=\left\{\begin{array}{c}
\hat{y}_{1} \\
\hat{y}_{2} \\
\hat{\theta} \\
\widehat{F}_{c}
\end{array}\right\}=\left[\begin{array}{ccccc}
c_{1,1} & c_{1,2} & \cdots & c_{1,2 n} & 0 \\
c_{2,1} & c_{2,2} & \cdots & c_{2,2 n} & 0 \\
c_{5,1} & c_{5,2} & \cdots & c_{5,2 n} & 0 \\
0 & 0 & \cdots & 0 & 1
\end{array}\right] \hat{x}_{e}=\boldsymbol{C}_{0(4 \times(2 n+1))} \hat{x}_{e((2 n+1) \times 4)}
$$

where $\hat{y}_{0}$ is defined as a physical output of the system which is estimated by Kalman filter algorithm and includes the estimation of the tool tip vibrations at point 1 and point $2\left(\hat{y}_{1}\right.$ and $\left.\hat{y}_{2}\right)$, tool tip bending coordinate $(\hat{\theta})$, and applied force $\left(\widehat{F}_{c}\right)$.

\subsection{Validation procedure}

The experimental validation of the designed Kalman-based observer was organized in two different sessions, as summarized in Fig. 6.

In the first sessions, the observer capability of compensating the machine tool dynamics was evaluated. The frequency bandwidth of the estimations was analyzed. For this purpose, series of pulse tests with instrumented hammers were conducted. The estimations performed by the designed observer were compared with the direct measurements. The apllied force was measured through the sensorized hammer while the tool tip displacements (point 1 and point 2) where measured by the inductive relative displacement sensors.

In the second part of the validation procedure real cutting tests (steel milling) were performed. In this case the observer estimations were compared with the measurements done by the inductive relative displacement sensor located at point 2 and the cutting force measurements performed through a Kistler dynamometer 9255B (sensitivity for $\mathrm{X}$ and $\mathrm{Y}$ direction $8 \mathrm{pC} / \mathrm{N}$ and $3.4 \mathrm{pC} / \mathrm{N}$ for $\mathrm{Z}$ direction) coupled to the charge amplifier $5070 \mathrm{~A}$ with the following setting: $1 \mathrm{mV} / \mathrm{N}$ for $\mathrm{X}$ and 
$\mathrm{Y}$ and $0.5 \mathrm{mV} / \mathrm{N}$ for $\mathrm{Z}$ direction. During cutting tests, the tool tip vibration measurement (point 1) was not feasible because the tool was engaged in the workpiece.

Estimation Properties Check

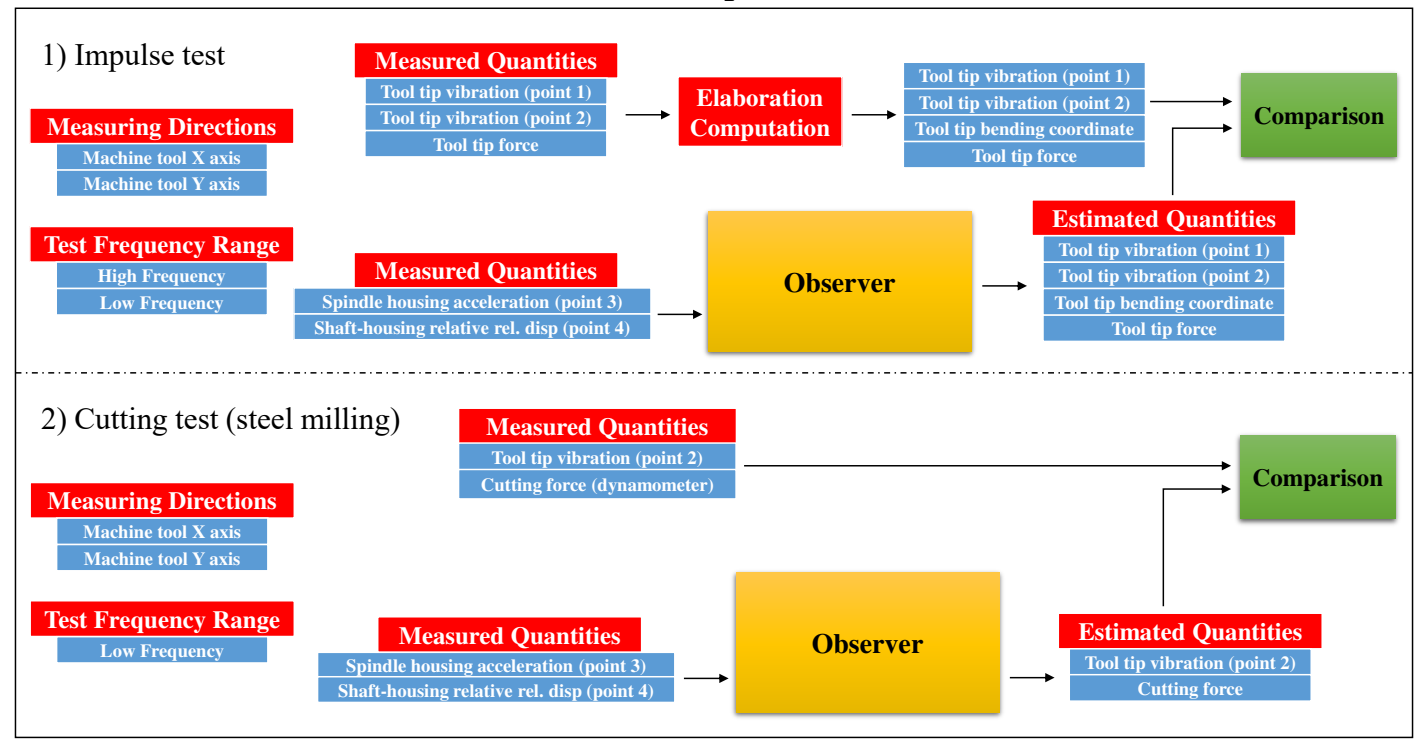

Fig. 6. Schematic representation of the experimental final validation.

\section{Results and discussion}

In order to accomplish the estimation process and to emphasize on the desired frequency ranges of the estimations, the Kalman gain matrix has to be tuned by allocating proper weights to the sensors data. This can be done by assigning appropriate quantities to process noise $w$ and measurement noise $z$ parameters. Several preliminary simulations that consider the plant model, the observer and the noise of the measurements were conducted in order to tune the Kalman filter gains. The tuning procedure has to be performed every time a different machine tool along with different integrated sensors are considered. For the considered case, the optimum results achieved after tuning the covariance matrix $\boldsymbol{Q}$ of the process noise and the covariance matrix $\boldsymbol{R}$ of the measurement noise are:

$$
\boldsymbol{Q}=4 \times 10^{23} ; \quad \boldsymbol{R}=\left[\begin{array}{cc}
1 & 0 \\
0 & 1.5 \times 10^{7}
\end{array}\right]
$$

In the following section, the estimation results are going to be compared and analyzed with the corresponding experimentally measured data according to the validation procedure already explained.

\subsection{Pulse tests}

In order to test the estimation capabilities of Kalman filter, pulse test were performed to excite the structural modes of the machine tool, as done also by Albertelli et al. in [24]. Estimated and experimentally measured quantities were compared in both $\mathrm{X}$ and $\mathrm{Y}$ directions and for low and medium-high frequency ranges. The low frequency pulse force can be used to emulate a series of generic low cutting speeds milling while, the high frequency excitation is representative of high-speed cutting operations. The hammer used for the low frequency test was a PCB 086D20 with soft rubber tip; whereas, in order to excite the medium-high frequency machine dynamics, a PCB 086D05 hammer with nylon tip was used.

Fig. 7 shows the comparison between the estimated and measured tool tip vibration at point. Both time and frequency domains are reported for each direction of excitation and for each excited frequency range. According to Fig. 7.a and Fig. 7.b, the low frequency estimation results show a good agreement with the measured values in both $\mathrm{X}$ and $\mathrm{Y}$ directions. Nonetheless, the estimation along Y direction seems to be a little less accurate compared to its almost flawless counterpart on $\mathrm{X}$ direction. The observer is able to estimate quite accurately the maximum vibration amplitude but exhibits some discrepancies in the estimation of the lowest frequency component. This could be due to a lack of model accuracy that is strictly related to the procedure (tap test) used to identify the model. Indeed, it is quite difficult to properly excite the low frequency machine dynamics using a hammer and, as a consequence, the identified model can't be very accurate. Nevertheless, the presented estimated results are good for the estimation in steel and aluminum milling. 
The presented frequency analysis shows the observer capability to compensate the machine dynamics over the frequency of interested. Low frequency tests were done to analyze the observer performance into compensating the mainly machine structure dynamics. When the same test was performed exciting the medium-high frequency range, the system behaves according to Figures 6.c and 6.d where the estimation results are reported as well. It is clearly observable that, as already pointed out for low frequencies, the estimation performed by the observer is quite accurate in both $\mathrm{X}$ and $\mathrm{Y}$ directions.

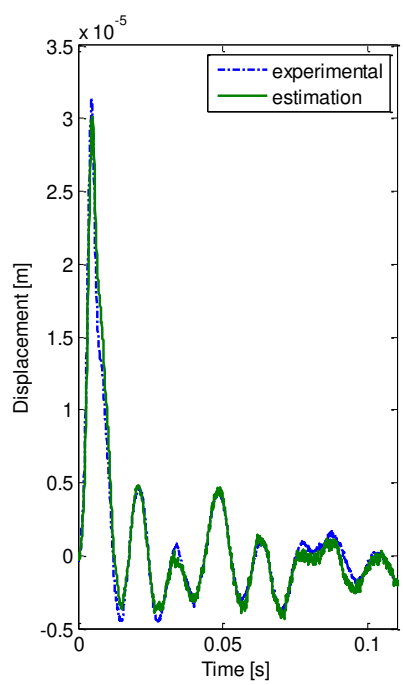

(a)

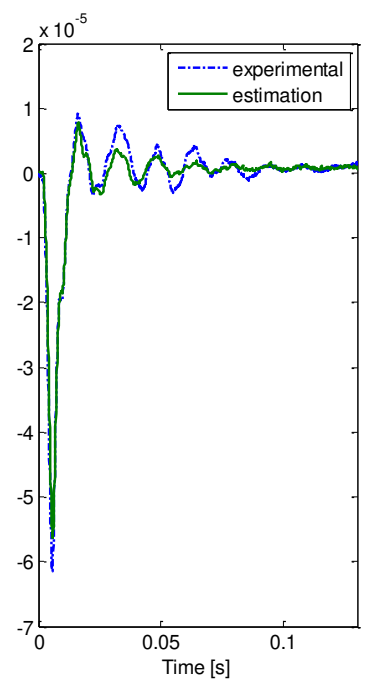

(c)

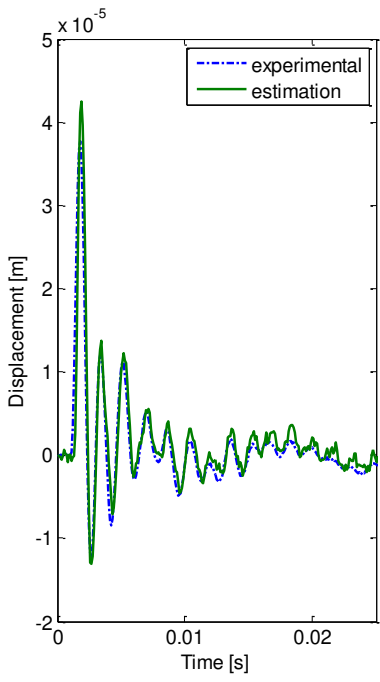

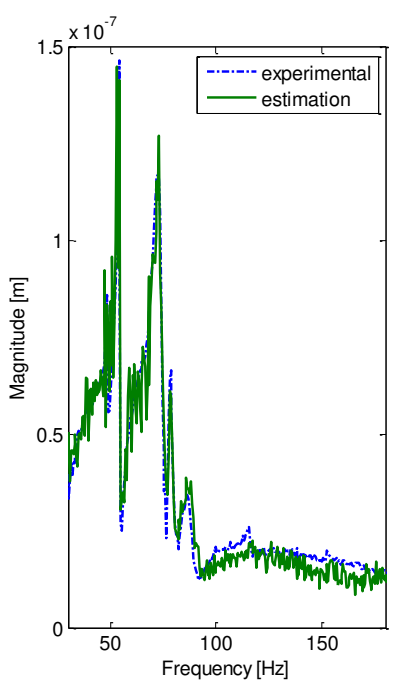

(b)

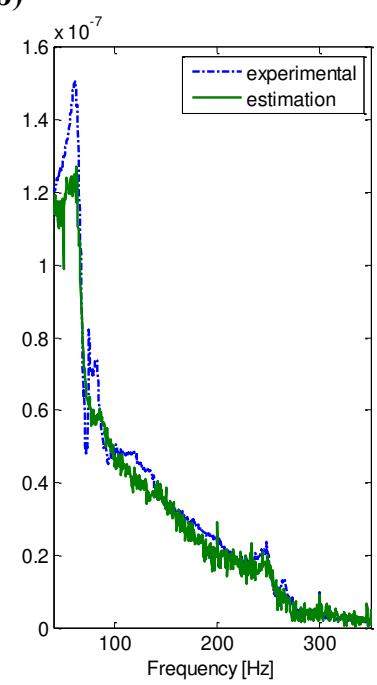

(d)

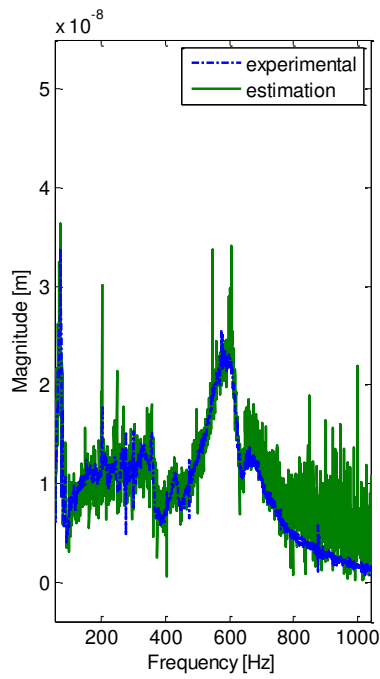

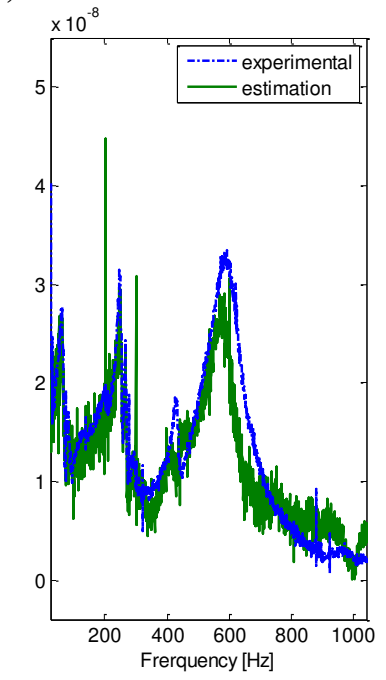

Fig. 7. Comparison between experimental and estimated machine vibrational responses at tool tip (point 1) to a force pulse. Time domain (a) and frequency domain (b) of the vibration in X (left) and Y (right) directions for low frequency excitation; time domain (c) and frequency domain (d) of the vibration in $\mathrm{X}$ (left) and $\mathrm{Y}$ (right) directions for medium-high frequency excitation.

In order to validate the observer capabilities during cutting operations, since point 1 would not be accessible anymore, a good estimation process is desirable for point 2 as well. Likewise, for bending coordinates evaluation, estimation of tool tip displacement at both point 1 and point 2 must be well assessed. Thus, observer estimating performances for tool vibration at point 2 are explored in Fig. 8. It can be observed that accuracy of the estimations remains unchanged. For both the low frequency range excitation (Fig. 8.a and Fig. 8.b) and the medium-high frequency range excitation (Fig. 8.c and Fig. 8.d), results are fully comparable with ones obtained for point 1 .

Moreover, for $\mathrm{X}$ direction, the comparisons between the measured and the estimated tool bending coordinates are reported in Fig. 9. Tool bending deflections were processed using the displacement measurements (point 1 and point 2) performed through the external inductive sensors, Fig. 2. Since the spindle exhibits its dynamics at medium-high frequencies, the accuracy of the comparison was evaluated in this frequency range. Even if the tool bending deflection estimation is not accurate as the results obtained for the tool displacement, the overall experimental-estimation agreement seems acceptable. As the vibration plays an important role on surface roughness, an on-line estimation of tool tip vibrations (displacement and bending deflection) could be utilized to determine the machined surface quality. This will be the object of further research works. 
(a)
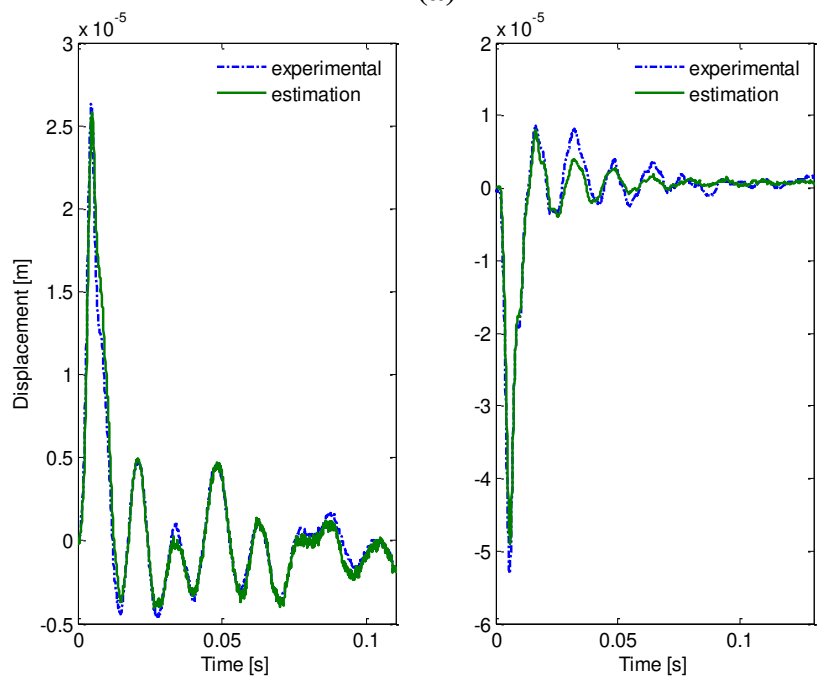

(c)

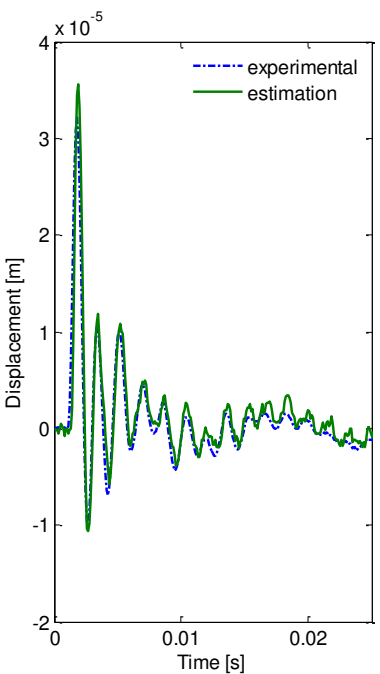

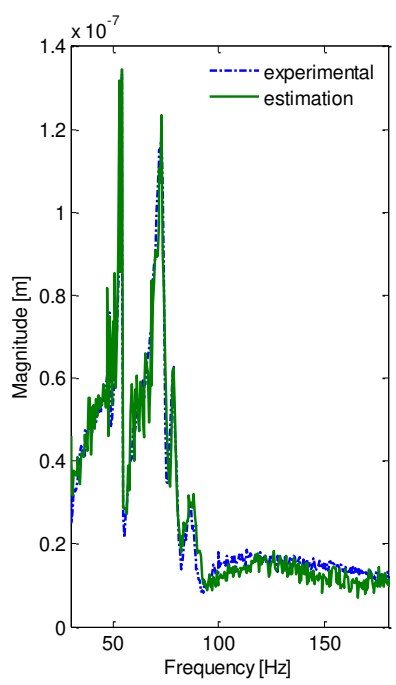

(b)

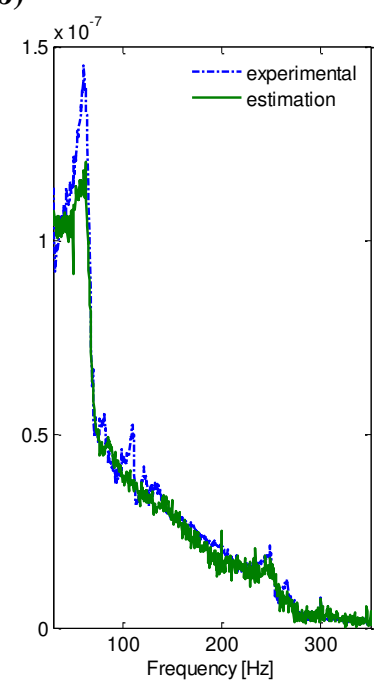

(d)

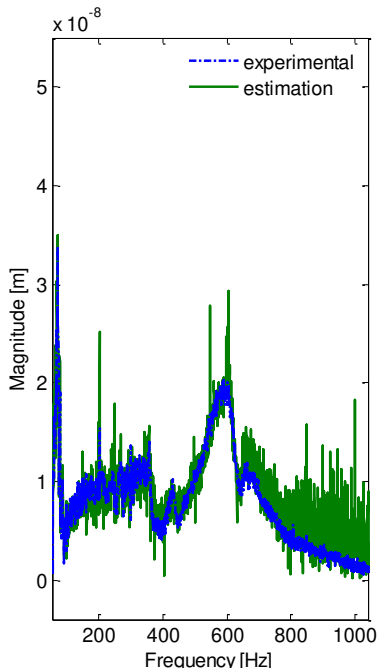

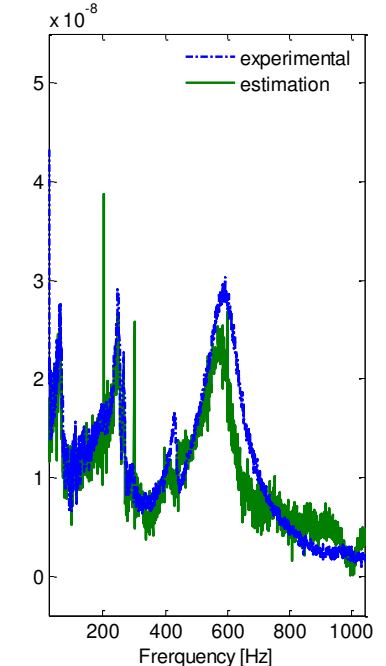

Fig. 8. Comparison between experimental and estimated machine responses at tool tip (point 2) for pulse force. Time domain (a) and frequency domain (b) of the vibration in X (left) and Y (right) directions for low frequency excitation; time domain (c) and frequency domain (d) of the vibration in $\mathrm{X}$ (left) and $\mathrm{Y}$ (right) directions for medium-high frequency excitation.
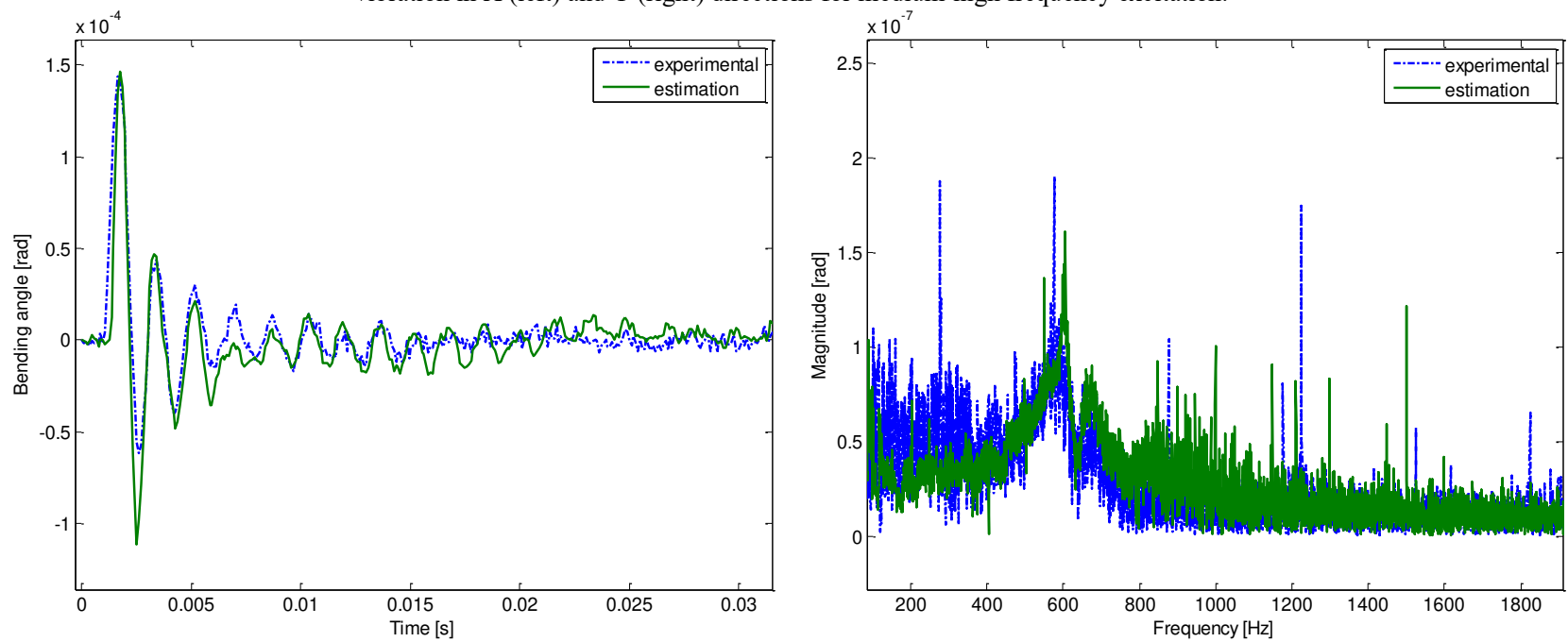

Fig. 9. Comparison between experimental and estimated tool tip bending response along $\mathrm{X}$ direction for high frequency pulse force. 
Finally, the observer capability of estimating the force applied to the tool can be appreciated in Fig. 10 where both the $\mathrm{X}$ and $\mathrm{Y}$ measurement-estimation comparisons are presented. The force applied to the tool was measured by means of the instrumented hammers used to perform this phase of the experimental validation. Only a slight oscillation can be observed in the force estimation. The oscillation is mainly due to small plant-model discrepancies. Indeed, at each step of the observer computation, the comparison between the sensors measurements and the linked estimations is used to update the state of the observer that includes also the unknown force applied to the tool tip. The observer interprets differences in the estimation of the vibrational behavior of the plant as forces applied to the tool tip. Nevertheless these small residual oscillations, the force applied to the tip was reasonably estimated.
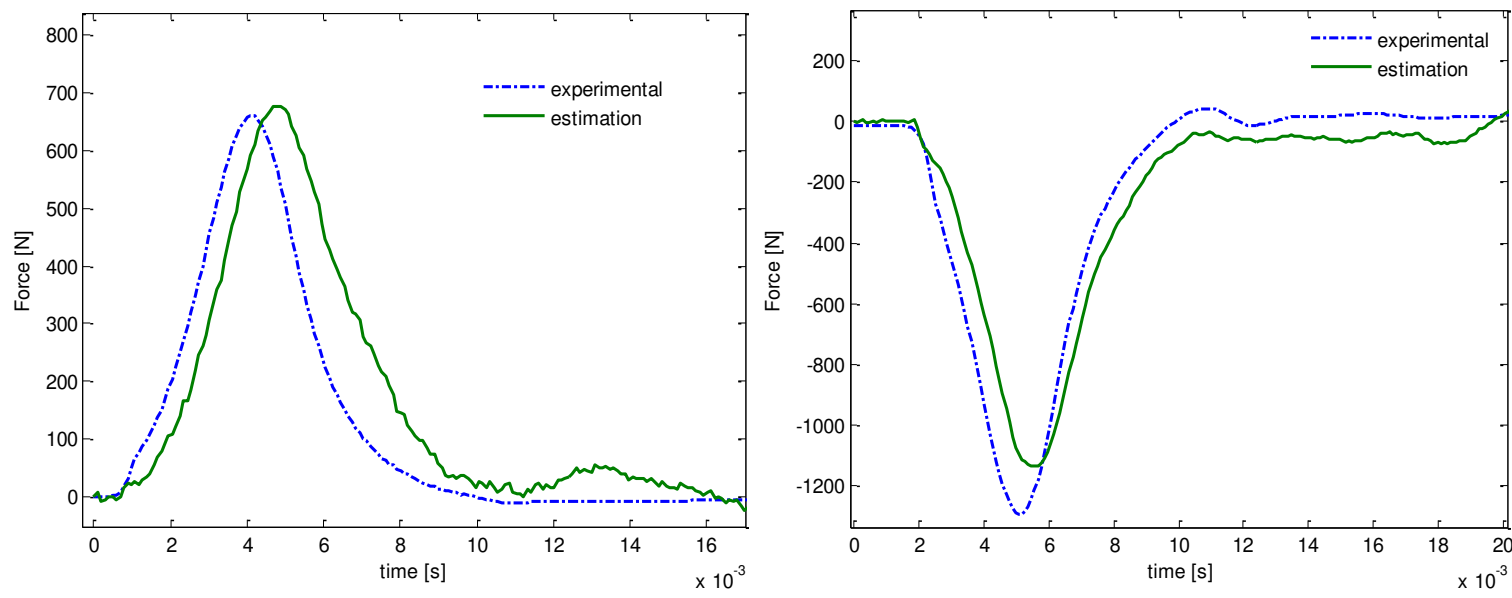

Fig. 10: Low frequency force pulse, $\mathrm{X}$ (left)/Y (right) direction, (experimentally measured - estimation)

\subsection{Cutting tests - steel milling}

As already explained, the designed observer was also tested during realistic milling operations.

The machined workpiece material is ISO C45 carbon steel. The tests were performed at $3.5 \mathrm{~mm}$ depth of cut, $915 \mathrm{rpm}$ spindle speed and $0.2 \mathrm{~mm} /$ tooth feed in dry full immersion configuration. During the tests the cutting forces were measured by a Kistler dynamometer 9255B while the tool vibrations were measured by the inductive sensors placed at point 2. Fig. 11 shows the comparisons between the estimated and measured quantities for cutting forces and tool tip displacements (point 2) in time domain. Both the early engagement phase and the regime phase along $\mathrm{X}$ direction are reported.
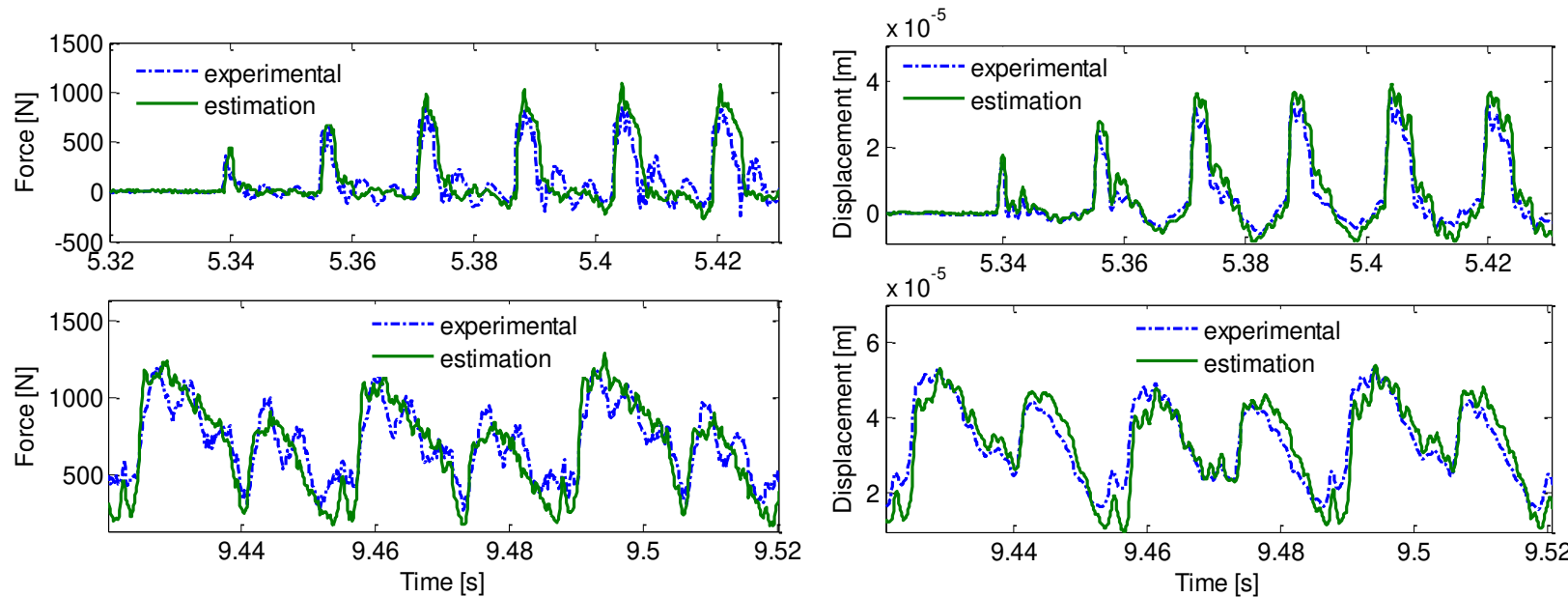

Fig. 11. Cutting force in X direction (left) during the first tool-workpiece engagement phase (above) and at the regime stage (below); tool tip vibration along $\mathrm{X}$ direction (right) during the first tool-workpiece engagement phase (above) and at the regime stage (below).

Looking at the pictures, the capabilities of the designed Kalman filter are fully confirmed. Starting from spindle housing vibration and spindle housing-shaft relative displacement, tool tip displacements and force components are very well estimated. Furthermore, even during a heavy discontinuous phase, i.e. the engagement phase, the estimation accuracy is high. Similar results (Y direction) are reported in Fig. 12. 

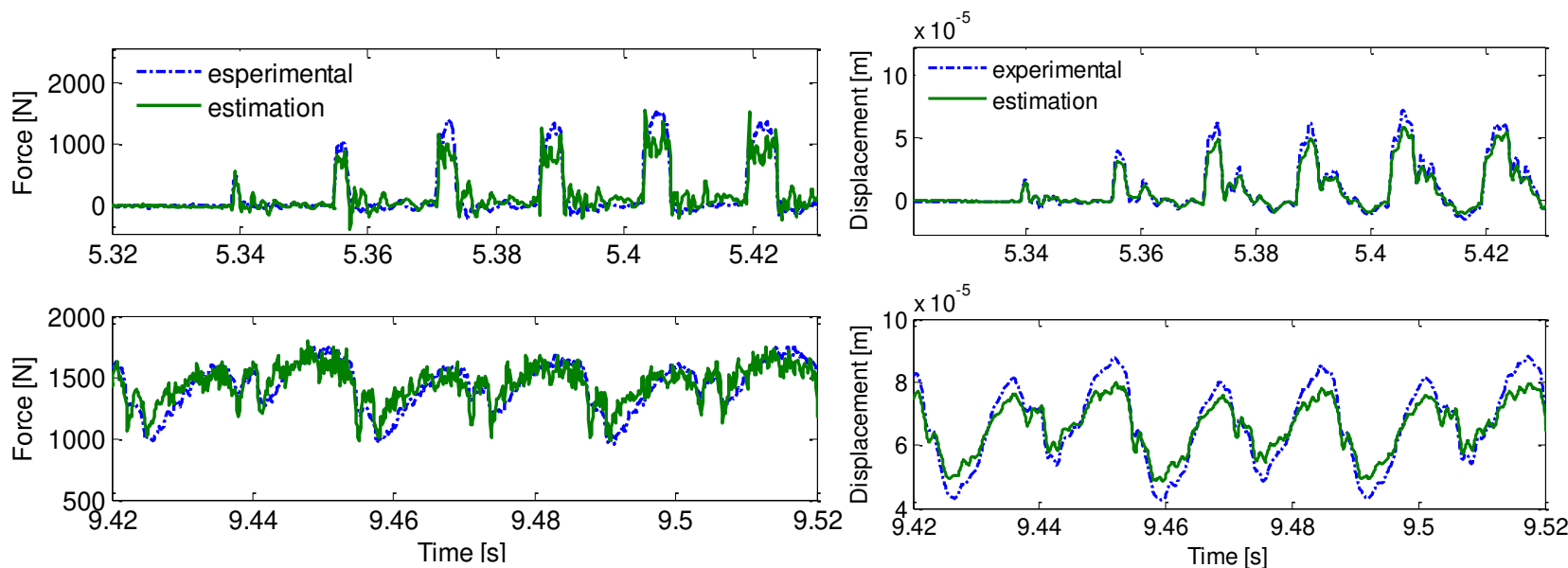

Fig. 12. Cutting force in Y direction (left) during the first tool-workpiece engagement phase (above) and at the regime stage (below); tool tip vibration along $\mathrm{Y}$ direction (right) during the first tool-workpiece engagement phase (above) and at the regime stage (below).

In order to better analyse the obtained results, the spectral analysis performed both on vibrations and on forces was reported in Fig. 13. It shows that the higher estimation errors (maximum 37\% both for forces and vibrations) are connected to the tooth passing frequency $(61 \mathrm{~Hz})$ components (mainly along $\mathrm{Y}$ direction). Other spectral components are characterized by much lower errors. This is mainly caused by the amplification effect connected to the machine tool structure eigenfrequencies. The identified model seems less reliable in the low frequency range. This is generally twofold. Firstly, the effect is typically caused by the adopted experimental procedure that does not allow to properly excite the low frequency machine tool resonance (characterized by high modal masses) with a sensorized hammer. Secondly, machine centres are often experimentally characterized in the stand still condition and not during a real machining operation. Both the aspects can be the origin of the revealed measured-estimated differences. Even if the limitations connected to the used machine tool experimental dynamic characterization procedure are quite known in literature, it still represents one of the most widespread adopted approach.

As can be observed in Fig. 13, the measured-estimation differences are more evident along Y direction due to a resonance close to the tooth passing frequency that amplifies the plant-model discrepancy. Even if differences can be observed in the performed estimations (mainly for the tooth passing frequency), the observer seems to be suitable for the conceived purpose that is the cutting forces and surface quality (through the tool tip vibration estimation) real-time monitoring. Compared to previous literature versions, the developed observer is able, exploiting also the accelerometer data, to roughly compensate the dynamic amplification (both for forces and vibrations) due to the low-frequency machine tool structure resonances.
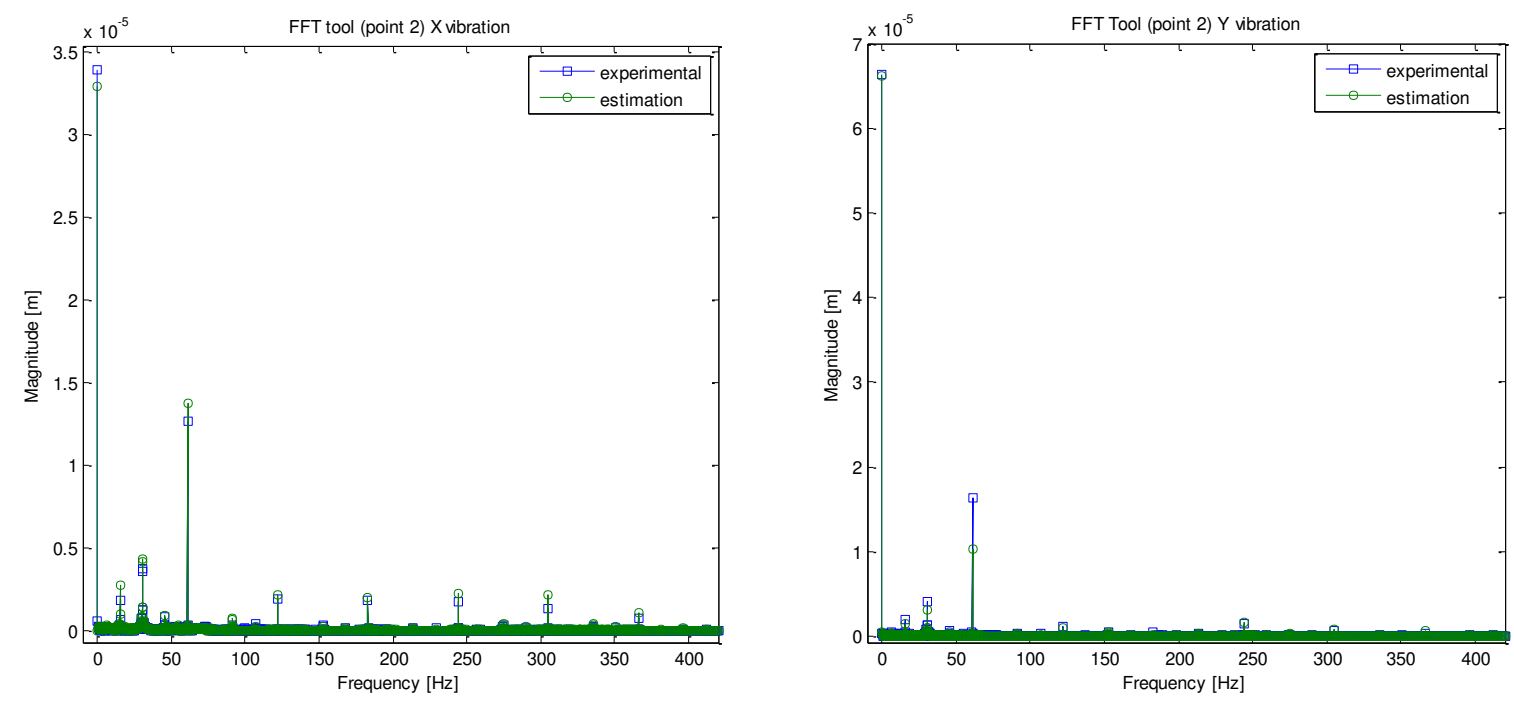


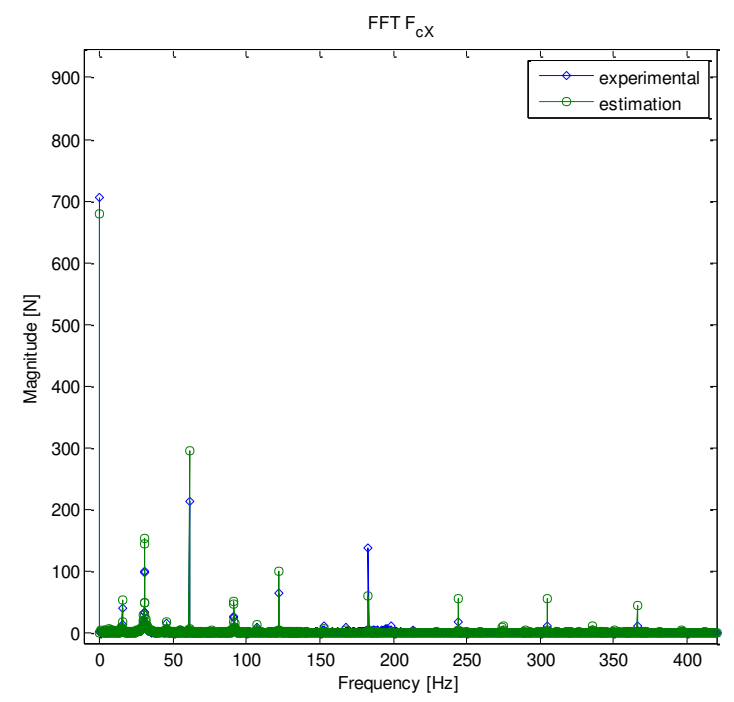

(c)

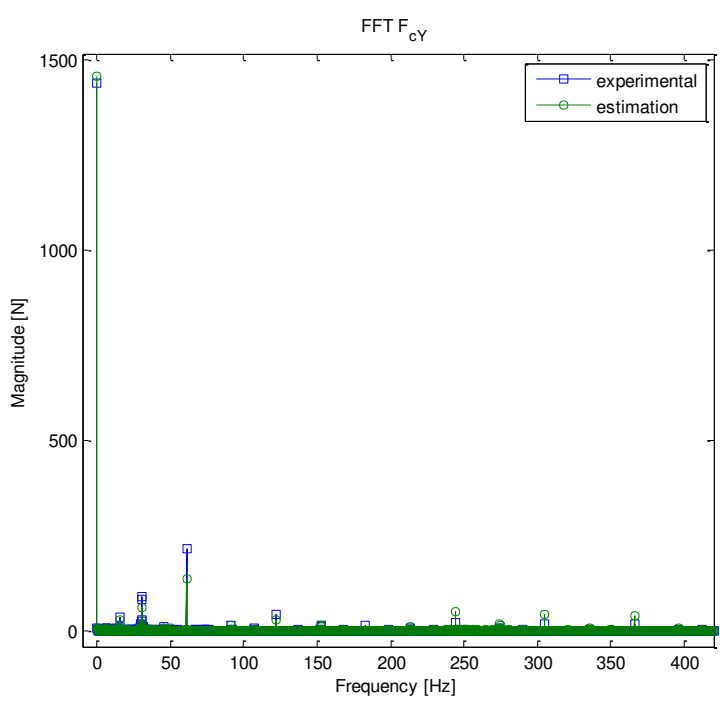

(d)

Fig. 13. Frequency domain. Experimental-estimation comparison: (a) tool tip vibration (point2) X direction, (b) tool tip vibration (point2) Y direction, (c) cutting force $\mathrm{X}$ direction and (d) cutting force $\mathrm{Y}$ direction.

In order to statistically analyse the prediction properties of the observer, the coherence function was computed for each coupled measured-estimated quantity. The coherence function (Eq. (28)) was evaluated both for tool vibrations and cutting forces along $\mathrm{X}$ and $\mathrm{Y}$ axes.

$$
\hat{\gamma}_{l m}(f)=\frac{\widehat{H}_{l m}(f) \widehat{H}_{m l}(f)}{\widehat{H}_{l l}(f) \widehat{H}_{m m}(f)}
$$

$\widehat{H}_{l m}(f)$ is the cross-spectrum estimation of the generic $l$ and $m$ signals whereas $\widehat{H}_{l l}(f)$ and $\widehat{H}_{m m}$ are respectively the autospectrum of the $l$ and $m$ signals. " $l$ " is the generic experimental measured quantity (tool vibration or force) whereas " $m$ " is the corresponding estimated quantity.

$\widehat{H}_{l m}(f), \widehat{H}_{l l}(f)$ and $\widehat{H}_{m m}(f)$ were estimated using the following relationships. As can be observed, each estimation was performed considering an averaging process that involved subsequent $\mathrm{n}_{\mathrm{d}}$ signal portions.

$$
\begin{gathered}
\widehat{H}_{l l}(f)=\frac{1}{n_{d}} \sum_{1}^{n_{d}} l^{*}(f) l(f) \\
\widehat{H}_{m m}(f)=\frac{1}{n_{d}} \sum_{1}^{n_{d}} m^{*}(f) m(f)
\end{gathered}
$$




$$
\widehat{H}_{l m}(f)=\frac{1}{n_{d}} \sum_{1}^{n_{d}} l^{*}(f) m(f)
$$

The estimated coherence functions were depicted in Fig. 14. As can be easily observed, the coherence functions exhibit quite high values (close to 1) up to $800 \mathrm{~Hz}$. This means that the developed observer, is able to compensate the machine tool dynamics for a quite extended frequency range. This confirms the considerations done analysing Fig. 7. Both for X and $\mathrm{Y}$ direction, the coherence functions connected to tool vibrations are higher that the coherence functions linked to the cutting forces.
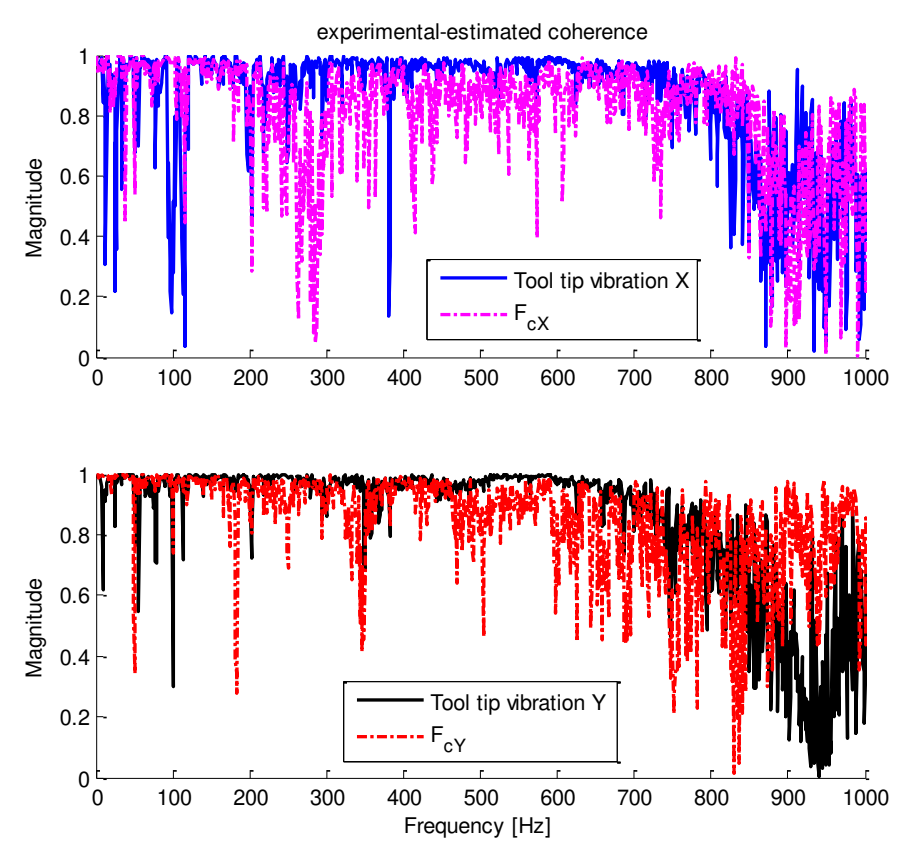

Fig. 14. Coherence Function experimental measurements - estimations. Tool tip vibration (point2) and cutting forces both along $\mathrm{X}$ and $\mathrm{Y}$ axes

In a similar way, the correlation coefficients $\boldsymbol{r}_{l m}(l, m)$ were computed as follows:

$$
\boldsymbol{r}_{l \boldsymbol{m}}(l, m)=\frac{\boldsymbol{c}(l, m)}{\sqrt{\boldsymbol{c}(l, l) \cdot \boldsymbol{c}(m, m)}}
$$

Where $\mathbf{c}$ is the covariance matrix. The computed correlation coefficients are reported in Table 3.

Table 3. Correlation coefficients experimental measurements - estimations. Tool tip vibration (point2) and cutting forces both along $\mathrm{X}$ and $\mathrm{Y}$ axes

\begin{tabular}{lll}
\hline Quantity & $\begin{array}{l}\text { Correlation coefficient } r_{l m} \\
\text { X direction }\end{array}$ & $\begin{array}{l}\text { Correlation coefficient } r_{l m} \\
\text { Y direction }\end{array}$ \\
\hline Tool tip vibration & 0.9022 & 0.98 \\
Force & 0.8813 & 0.7842 \\
\hline
\end{tabular}

The highest correlation coefficient (close to 1) can be revealed for tool tip vibration along Y direction. According to the analysis performed on the coherence functions, the correlation coefficients connected to vibrations signals are higher than the coefficients linked to forces. 
As already observed, notwithstanding the little revealed inaccuracy on some frequency components, the Kalman-based observer is proved to be a valid instrument to assess real cutting conditions and it open novel opportunities in the monitoring of both the process and the quality of the processed part.

Compared to previous research works (e.g. [9] and [10]), the achieved improvements are twofold.

Firstly, the conceived observer allows estimating the tool tip vibrations that is a useful information strictly connected to the surfaces quality of the processed workpiece. Secondly, thanks to a multi-sensors approach, the frequency bandwidth is extended especially in the low frequency range. An accelerometer that is a low-priced sensor was placed on the spindle housing to allow compensating the low frequency resonances connected to the machine tool structure dynamic behavior. This feature is particularly valuable when low-speed machining operations are performed. Therefore, the developed observer, compared to previous versions, can be reliably used over a broader range of working conditions corresponding to different processed materials. If significantly different tools (in terms of weight, stiffness and geometry) are mounted on the spindle, the observer needs to be re-characterized and a new experimental modal analysis session has to be performed. Since the experimental modal analysis is a quite time consuming activity, the procedure could be industrially feasible only if was applied to a limited number of tools. This is not far from the real needs because only for some critical milling operations it is meaningful to real time monitoring the cutting forces and the tool tip vibrations. Moreover, future researches will be focused on the exploitation of substructure analysis (i.e. [25] and [26]) techniques to fast update the machine dynamic model when different tools are used.

\section{Conclusions}

This paper presents a novel methodology for the in-process indirect estimation of cutting forces and tool tip vibrations (displacement and bending deflection) in milling operations. Starting from the dynamical model of the tool-spindlemachine system, a Kalman-based observer was designed. The observer processes the spindle housing accelerations and the spindle shaft-housing relative displacements. Sensors fusion made the estimation frequency bandwidth wider and thus made the observer suitable for different milling operations. The observer capabilities were verified through experimental tests. In the first validation section, the accuracy of the observer estimation was analyzed performing pulse force tests. In the second section, real cutting tests were performed. In both the experimental sections, a good agreement between the measurements and the estimation was achieved. Future works will be focused on the analysis of the observer robustness and on the exploitation of substructure analysis (i.e. [25] and [26]) techniques to fast update the machine dynamic model when different tools are used. Moreover, future researches will deal with the exploitation of the vibrations estimation for a more direct surface quality evaluation as reported in [18].

\section{Acknowledgements}

The authors strongly acknowledge MUSP consortium, MANDELLI Sistemi and Capellini Electrospindle that provided respectively the laboratory facilities, the machine tool and the electrospindle for the tests. Moreover, the authors would thank "Progetto Giovani Ricercatori - mechanical engineering department - Politecnico di Milano" and the national project "CLUSTER - La fabbrica Intelligente - High Performance Manufacturing - Ministero dello Sviluppo Economico CTN01_00163_216758" that provided the financial and scientific framework for the present research.

\section{Compliance with Ethical Standards}

The authors of this research do not have any conflicts of interest. The research does not involve Human participants or animals and the authors warrant that the paper fulfills with the ethical standards of the journal.

\section{References}

[1] Andrews GC, Tlusty J (1983) A critical review of sensors for unmanned machining. CIRP Annals - Manufacturing Technology 32 (2) $563-572$

[2] Byrne G, Dornfeld D, Inasaki I, Ketteler G, Konig W, Teti R (1995) Tool condition monitoring (TCM) - the status of research and industrial application. CIRP Annals - Manufacturing Technology 44 (2): 541-567

[3] Tonshoff HK, Wulfsberg JP, Kals HJJ, Konig W, van Luttervelt CA (1998) Development and trends in monitoring and control of machining process. CIRP Annals - Manufacturing Technology 37 (2): 611-622

[4] Lee P, Altintas Y (1996) Prediction of ball end milling forces from orthogonal cutting data. International Journal of Machine Tools and Manufacture 36 (9): 1059-1072

[5] Teti R, Jawahir IS, Jemielniak K, Segreto T, Chen S, Kossakowska J (2006) Chip form monitoring through advanced processing of cutting force sensor signals. CIRP Annals - Manufacturing Technology 55(1): 75-80

[6] Teti R, Jemielniak K, O’Donnell G, Dornfeld D (2010) Advanced monitoring of machining operations. CIRP Annals - Manufacturing Technology 59 (2): 717-739 
[7] Smith DA, Smith S, Tlusty J (1998) High performance milling torque sensor. Journal of Manufacturing Science and Engineering 120(3) 504-551

[8] Aoyama H, Inasaki I, Suda I, Ohzeki H (1998) Prediction of tool wear and tool failure in milling by utilizing magnetorestrictive torque sensor. Technical papers of the north American Manufacturing Research Institution of SME, pp 125-130

[9] Albrecht A, Park SS, Altintas Y, Pritschow (2005) High frequency bandwidth cutting force measurement in milling using capacitance displacement sensors. International Journal of Machine Tools and Manufacture 45 (9): 993-1008

[10] Park SS, Altintas Y (2004) Dynamic compensation of spindle integrated force sensors with Kalman Filter, International Journal of Dynamic Systems, Measurement, and Control 126(3): 443-452

[11] Kim JH, Chang HK, Han DC, Jang DY (2005) Cutting force estimation by measuring spindle displacement in milling process. CIRP Annals Manufacturing Technology 54 (1): 67-70

[12] Sarhan AD, Matsubara A, Sugihara M, Saraie H, Ibaraki S, Kakino Y (2006) Monitoring method of cutting force by using additional spindle sensors. JSME International Journal Series C: 307-31

[13] Tonshoff HK, I. Inasaki I (2001) Sensors in manufacturing. Wiley-VCH, ISBN 3-527r-r29558-5.

[14] Chae J, Park SS (2007) High frequency bandwidth measurements of micro cutting forces. International Journal of Machine Tools and Manufacture 47 (9): 1433-1441

[15] Salehi M, Albertelli P, Goletti M, Ripamonti F, Tomasini G (2014) Indirect model based estimation of cutting force and tool tip vibrational behaviour in milling machines by sensor fusion. $9^{\text {th }}$ CIRP Conference on Intelligent Computation in Manufacturing ICME, Naples (Italy).

[16] Möhring HC, Litwinski KM, Gümmer O (2010) Process monitoring with sensory machine tool components. CIRP Annals - Manufacturing Technology 59 (1): 383-386

[17] Denkena B, Hackelöer FL (2010) Multi-sensor disturbance force measurement for compliant mechanical structures. 9th Annual IEEE Conference on Sensors pp 2518-2524, Seoul

[18] Jiang H, Long X, Meng G (2008) Study of the correlation between surface generation and cutting vibrations in peripheral milling. Journal of Material Processing Technology 208 (1-3): 229-238

[19] Montgomery D, Altintas Y (1991) Mechanism of cutting force and surface generation in dynamic milling. Journal of Manufacturing Science and Engineering 113(2): 160-168

[20] Paris H, Peigne G, Mayer R (2004) Surface shape prediction in high speed milling. International Journal of Machine Tools and Manufacture 44 (15): 1567-1576

[21] Peigne G, Paris H, Brissaud D, Gouskov A (2004) Impact of the cutting dynamics of small radial immersion milling operations on machined surface roughness. International Journal of Machine Tools and Manufacture 44 (11):1133-1142

[22] Cheung CF, Lee WB (2000) Modelling and simulation of surface topography in ultra-precision diamond turning. Journal of Engineering Manufacture 214(6): 463-480

[23] Albertelli P, Cau N, Bianchi G, Monno M (2012) The effect of dynamic interaction between machine tool subsystems on cutting process stability. The International Journal of Advanced Manufacturing Technology 58 (9-12): 923-932

[24] Albertelli P, Elmas S, Jackson MR, Bianchi G,Parkin RM, Monno M (2012) Active Spindle System for a Rotary Planing Machine. The International Journal of Advanced Manufacturing Technology 63: 1021-1034

[25] Albertelli P, Goletti M, Monno M (2013) A new Receptance Coupling Substructure Analysis methodology to improve Chatter Free cutting conditions prediction. International Journal of Machine Tool Manufacture 72: 16-24

[26] Movahhedy MR, Gerami JM (2006) Prediction of spindle dynamics in milling by sub-structure coupling. International Journal of Machine Tool and Manufacture 46: 243-251 